\title{
Some Methods of Estimation from Censored Samples in Exponential and Gamma Models
}

\author{
R R. L Kantam \\ Acharya Nagarjuna University, Guntur, India, kantam.rrl@gmail.com \\ B Sriram \\ ANU College of Engineering and Technology, Guntur, India, sriram_stat@rediffmail.com
}

Follow this and additional works at: http://digitalcommons.wayne.edu/jmasm

Part of the Applied Statistics Commons, Social and Behavioral Sciences Commons, and the Statistical Theory Commons

\section{Recommended Citation}

Kantam, R R. L and Sriram, B (2014) "Some Methods of Estimation from Censored Samples in Exponential and Gamma Models," Journal of Modern Applied Statistical Methods: Vol. 13 : Iss. 2 , Article 17.

DOI: $10.22237 /$ jmasm/1414815360

Available at: http://digitalcommons.wayne.edu/jmasm/vol13/iss2/17 


\section{Some Methods of Estimation from Censored Samples in Exponential and Gamma Models}

\author{
R. R. L. Kantam \\ Acharya Nagarjuna University \\ Guntur, India
}

\author{
B. Sriram \\ ANU College of Engineering \& Technology \\ Guntur, India
}

Two popular life testing models exponential and one where its generalization is gamma are considered. Estimation of scale parameter from a general Type-II doubly censored sample is attempted by the principle of maximum likelihood method. Resulting equations found to be giving iterative solutions. As an alternative to iterative solution certain admissible modifications to the estimating equations are suggested in special cases. The resulting estimates are compared with the exact maximum likelihood estimates analytically or through simulation. The results are also extended for reliability estimation.

Keywords: Maximum likelihood estimation, Type-II doubly censored sample, order statistics, modified maximum likelihood estimate, asymptotic variance, BLUE, left censored sample, reliability estimation

\section{Introduction}

Exponential distribution is the distribution of a continuous life time random variable and is the focal distribution of any reliability study just as normal distribution in classical statistical inference. The gamma distribution is an extension of exponential distribution and its shape parameter $\theta$ is more than one. Such an IFR model specifying $\theta=2$ is considered so that the resulting density is a weighted exponential distribution/length biased version of exponential distribution. The probability density function, the cumulative distribution function, the reliability function, the failure rate of such a distribution are respectively

$$
\begin{gathered}
f(z)=z e^{-z} \\
F(z)=1-e^{-z}(1+z)
\end{gathered}
$$

R. R. L. Kantam is a professor in the Department of Statistics. Email him at kantam.rrl@gmail.com. B. Sriram is in the Department of Science \& Humanities. Email himatsriram_stat@rediffmail.com. 


$$
\begin{gathered}
R(z)=e^{-z}(1+z) \\
h(z)=\frac{z}{1+z}
\end{gathered}
$$

If a scale parameter $\sigma$ is introduced these functions become respectively

$$
\begin{gathered}
f(x)=\frac{x}{\sigma^{2}} e^{-\frac{x}{\sigma}} \\
F(x)=1-e^{-\frac{x}{\sigma}}\left(1+\frac{x}{\sigma}\right) \\
R(x)=e^{-\frac{x}{\sigma}}\left(1+\frac{\mathrm{x}}{\sigma}\right) \\
h(x)=\frac{x}{\sigma\left(1+\frac{\mathrm{x}}{\sigma}\right)}
\end{gathered}
$$

Generally the life time sample data are generated through a life testing experiment of a sample items giving rise to observations as soon as a sample items fail. The observations flow in a natural order giving rise to ordered sample data (the weaker items fail earlier, stronger items later). In view of the nature of a life testing experiment, it is possible to sometimes have an incomplete data with missing observations on either extremes of the sample - because of very early failures before data collection itself or because some items not failing at all even after a long waiting. Such a sample is called a doubly Type II censored sample. If $n$ items are originally put to test in which it was not possible to have data on lives of the weakest $r_{1}$ items, strongest $r_{2}$ the sample would be as follows

$\left[r_{1}\right.$ missing $] x_{r_{1}<} x_{r_{1}+1<\ldots \ldots . .<} x_{n-r_{2}} \quad\left[r_{2}\right.$ missing $]$

$1,2,3 \ldots \ldots . . r_{1}, r_{1}+1 \ldots \ldots \ldots \ldots \ldots . . n-r_{2}, n-r_{2}+1, \ldots \ldots . n$ 
This paper deals with the problem of estimation of $\sigma$ and reliability function $R(x ; \sigma)$ of a gamma distribution from a doubly Type II censored samples.

Estimation of $\sigma$ in a gamma distribution using the well-known ML method is attempted by many researchers from complete as well as censored samples. Whenever a particular method of estimation is resulting in iterative solutions as values of the estimator in any density some admissible adhoc modifications are suggested to that method of estimation by many researchers in order to overcome iterative solution and get a reasonably efficient estimator. In this context Tiku (1967), Mehrotra and Nanda (1974), Cohen and Whitten (1982), Balakrishanan and Cohen (1990), Tiku and Suresh (1992), Rosaiah et al. (1993a), Rosaiah et al. (1993b), Rosaiah et al. (1997), Kantam and Srinivasa Rao (2002), Srinivasa Rao and Kantam (2004), Kantam et al. (2007), Rosaiah et al. (2007) and the references therein are some instances.

Motivated by the modified methods of estimation reported in the literature some modifications are proposed to estimate the scale parameter $\sigma$ of gamma distribution from a doubly Type II censored sample starting with the well-known maximum likelihood method of estimation. Later modified estimates are used to obtain the reliability estimates also, in addition an attempt is made to establish the asymptotic equivalence of the proposed modifications. Small sample comparisons are also presented. Results of exponential distribution are also given. The findings about estimation of $\sigma$ are described in the following section and those of reliability estimation are given in the section with that heading.

\section{Parametric Estimation}

Let $X_{r_{1}+1}<X_{r_{1}+1}<\ldots \ldots . .<X_{n-r_{2}}$ be a doubly Type II censored sample from a scale gamma distribution out of an originally planned random sample of size $n$ in which the least $r_{1}$ observations, the largest $r_{2}$ observations are missing. The likelihood function of such a sample is given by

$$
L \alpha\left[F\left(x_{r_{1}+1}\right)\right]^{r_{1}}\left[\prod_{i=r_{1}+1}^{n-r_{2}} f\left(x_{i}\right)\right]\left[1-F\left(x_{n-r_{2}}\right)\right]^{r_{2}}
$$

Where $f(),. F($.$) are given in Equations (5), (6). Substituting the relevant expressions,$ taking logarithms, differentiating with respect to $\sigma$ and equating to zero, after some simplifications, results in 


$$
\frac{-r_{1} z_{r_{1}+1}^{2} e^{-z_{r_{1}+1}}}{1-\left(1+z_{r_{1}+1}\right) e^{-z_{r_{1}+1}}}-2\left(n-r_{1}-r_{2}\right)-\sum_{i=r_{1}+1}^{n-r_{2}} z_{i}-\frac{r_{2} z_{n-r_{2}}^{2}}{1+z_{n-r_{2}}}=0
$$

Where $\mathrm{z}_{\mathrm{i}}=\frac{x_{i}}{\sigma}, i=r_{1}+1, \ldots . . n-r_{2}$.

It can be observed that Equation (10) must be solved iteratively to obtain the MLE of $\sigma$. However the complete sample situation $\left(\mathrm{r}_{1=} \mathrm{r}_{2}=0\right)$ gives $\sigma^{\wedge}=\frac{\bar{x}}{2}$. The failure censored sample situation ( $r_{1}=0, r_{2}=\mathrm{r}$ ) simplifies Equation (10) to the form

$$
A \sigma^{2}+B \sigma+C=0=
$$

$$
\text { where } \left.\begin{array}{rl}
\mathrm{A} & =2(\mathrm{n}-\mathrm{r}) \\
\mathrm{B} & =2(\mathrm{n}-\mathrm{r}) \mathrm{x}_{\mathrm{n}-\mathrm{r}}-\sum_{i=1}^{n-r} x_{i} \\
\mathrm{C} & =\sum_{\mathrm{i}=1}^{\mathrm{n}-\mathrm{r}} \mathrm{x}_{\mathrm{i}} \cdot x_{n-r}+r \cdot x^{2}{ }_{n-r}
\end{array}\right\}
$$

It can be proved that the quadratic Equation (11) will have only one positive root and hence is the maximum likelihood estimate of $\sigma$, which is

$$
\sigma^{\wedge}=\frac{-B+\sqrt{B^{2}-4 A C}}{2 A}
$$

The exact variance of MLE in Equation (13) cannot be obtained analytically and has to be computed only through simulation and the results were given in Table 4 (all Tables can be found in Appendix A). The asymptotic variance is given by formula

$$
\operatorname{asvar}\left(\sigma^{\wedge}\right)=\left[-E\left(\frac{\partial^{2} \log L}{\partial \sigma^{2}}\right)\right]^{-1}
$$


The left censored sample situation $\left(r_{1}=r, r_{2}=0\right)$ is of some interest. The estimating equation in this situation becomes

$$
\frac{-r z_{r+1}^{2} e^{-z_{r+1}}}{1-\left[1+z_{r+1}\right] e^{-z_{r+1}}}-2(n-r)-\sum_{i=r+1}^{n} z_{i}=0
$$

This equation cannot be solved for $\sigma$ analytically because of the structure of the term $\frac{r z_{r+1}^{2} e^{-z_{r+1}}}{1-\left[1+z_{r+1}\right] e^{-z_{r+1}}}$ corresponding to the left censored portion of the sample. Using the fact that $\frac{x_{i}}{\sigma}=z_{i}$ and Equations (5), (6) results in

$$
\frac{r . z_{r+1}^{2} e^{-z_{r+1}}}{1-\left[1+z_{r+1}\right] e^{-z_{r+1}}}=r \cdot z_{r+1} \frac{f\left(z_{r+1}\right)}{F\left(z_{r+1}\right)}
$$

Unless the portion given by (16) of the Equation (15) is approximated by a reasonable simpler expression, Equation (15) will not give analytical solution for $\sigma$ to get the MLE from a left censored sample. Similar approximations and modifications are suggested by many authors in many densities earlier. Among such works Tiku (1967) Balakrishanan and Cohen (1990, Ch.6), Tiku and Suresh (1992) are of relevance in the present investigation. Apart from these, specifically for gamma distribution, Mehrotra and Nanda (1974) suggested that the expression in (16) be approximated by its expected value to get simpler estimates of $\sigma$. Their approximation resulted in the well-known trimmed mean adjusted for unbiasedness as the approximate maximum likelihood estimator of $\sigma$.

The commonness in the modifications of Tiku (1967), Balakrishnan and Cohen (1990) and Tiku and Suresh (1992) is that they have approximated the expression $\frac{f\left(z_{r+1}\right)}{F\left(z_{r+1}\right)}$ of Equation (16) by a linear expression of the form $\alpha+\beta z$ (say), where $\alpha$ and $\beta$ are to be suitably found. This investigation assesses the strength of linearity of the two functions, namely

$$
h_{1}(z)=\frac{f\left(z_{r+1}\right)}{F\left(z_{r+1}\right)}=\frac{z \cdot e^{-z}}{1-e^{-z}(1+z)}
$$




\section{KANTAM \& SRIRAM}

and

$$
h_{2}(z)=\frac{z_{r+1} f\left(z_{r+1}\right)}{F\left(z_{r+1}\right)}=\frac{z^{2} \cdot e^{-z}}{1-e^{-z}(1+z)}
$$

in neighborhoods of $r+1^{\text {th }}$ quantile of the population. Let $p=\frac{r+1}{n+1}$, define $f\left(\xi_{r+1}\right)=\frac{r+1}{n+1}$ so that $\xi_{r}$ is the $r^{\text {th }}$ quantile. Let

$$
\left.\begin{array}{l}
P^{\prime}=p-\sqrt{\frac{p q}{n}} \\
P^{\prime \prime}=p+\sqrt{\frac{p q}{n}}
\end{array}\right\}
$$

Where $q=1$-p. Let $\left(z^{\prime}, z^{\prime \prime}\right)$ be the solutions of the equations $F\left(z^{\prime}\right)=P^{\prime}$ and $F\left(z^{\prime \prime}\right)=$ $P^{\prime}$ where $F(z)$ is given by Equation (6). Thus $\left(z^{\prime}, z^{\prime \prime}\right)$ is an interval in which $\xi_{r+1}$ is expected to fall. For this purpose the values of $F(z)$ for $z=0(0.01)$ to 11.89 have been tabulated and these are given in Table 1. For each combination of $n=3(1) 10$, $r=1,2, \ldots . n-2$, identified from Table 1 the values of $z$ close to $F\left(z^{\prime}\right), F\left(z^{\prime \prime}\right)$ and supposed to contain the interval $\left(z^{\prime}, z^{\prime \prime}\right)$. For example, when $n=5, r=1$ :

$$
\begin{gathered}
p=\frac{1}{3}, q=\frac{2}{3} \\
p-\sqrt{\frac{p q}{n}}=0.1225, \quad p+\sqrt{\frac{p q}{n}}=0.54413 \\
F\left(z^{\prime}\right)=0.1225, \quad F\left(z^{\prime \prime}\right)=0.54413
\end{gathered}
$$

From Table 1, search for the values of $F(\mathrm{z})$ closest to 0.1225 and less than 0.1225 , closest to 0.54413 and more than 0.54413 . In Table 1 when $z=0.6, F(z)=0.121901$, when $z=0.61, F(z)=0.125205$ we take $z^{*}=0.6$. Similarly when $z=1.82, F(z)=$ 0.543087 , when $z=1.83 F(z)=0.54603$. We take $z^{* *}=1.83$. By the non-decreasing property of distribution function the interval $\left(z^{\prime}, z^{\prime \prime}\right)$ falls in the interval $\left(z^{*}, z^{* *}\right)$.

Table 1 was utilized to obtain the intervals of the type $\left(z^{*}, z^{* *}\right)$ for $n=3(1) 10$ $r=1,2, \ldots n-2$. The functions $h_{1}(z)$ and $h_{2}(z)$ given by Equations (17), (18) respectively are then evaluated at various values of $z$ over $\left(z^{*}, z^{* *}\right)$. The $\operatorname{Karl}$ 
Pearson's product moment correlation coefficient is computed between $\left(z, h_{1}(z)\right)$, $\left(z, h_{2}(z)\right)$ for $z \in\left(z^{*}, z^{* *}\right)$ with the chosen combinations of $n \& r$. These are given in Table 2.

From Table 2 it may be observed that $\rho_{2}$ is larger than $\rho_{1}$ uniformly. Therefore it may be concluded that linearization of $h_{2}(z)$ is preferable to linearization of $h_{1}(z)$ for $z \in\left(z^{*}, z^{* *}\right)$. Because $\left(z^{\prime}, z^{\prime \prime}\right)$ is subset of $\left(z^{*}, z^{* *}\right)$ we approximate the expression

$$
h_{2}\left(z_{r+1}\right)=\frac{e^{-z_{r+1}} z_{r+1}^{2}}{1-\left(1+z_{r+1}\right) e^{-z}}
$$

of Equation (16) by a linear equation.

i.e.,

$$
\frac{e^{-z}{ }_{r+1} z_{r+1}^{2}}{1-\left(1+z_{r+1}\right) e^{-z_{r+1}}} \cong \alpha+\beta z_{r+1}
$$

where $\alpha$ and $\beta$ are to be suitably found. Substituting (21) in (15) and solving it for $\sigma$ results in the modified maximum likelihood estimate of $\sigma$ from left censored sample as

$$
\sigma^{\wedge}=\frac{\left[\sum_{i=r+2}^{n} x_{i}+(1-r \beta) x_{r+1}\right]}{[r \alpha+2(n-r)]}
$$

This is a linear estimator and its variance can be found using the moments and cross moments of standard ordered gamma statistics given by Prescott (1974), Balasooriya (1992), provided values of $\alpha$ and $\beta$ are known. Three different methods for calculating $\alpha, \beta$ are now considered. The basic works relevant to these three methods are - Tiku (1967), Balakrishnan and Cohen (1990, Ch.6), Tiku and Suresh (1992). The methods of getting $\alpha$ and $\beta$ are described using these works.

Here it should be reemphasized that linearization by earlier authors is for $\mathrm{h}_{1}(\mathrm{z})$, whereas the linearization herein is for $h_{2}(z)$, because $h_{2}(z)$ was found to be more linear than $h_{1}(z)$ as evidenced from sample Table 2. 


\section{KANTAM \& SRIRAM}

\section{Method I:}

Consider the equations $F\left(z^{\prime}\right)=P^{\prime}, F\left(z^{\prime \prime}\right)=P^{\prime \prime}$ where $p=\frac{r+1}{n+1}, \mathrm{q}=1-\mathrm{p}, \mathrm{P}^{\prime}=p-\sqrt{\frac{p q}{n}}$, $\mathrm{P}^{\prime \prime}=p+\sqrt{\frac{p q}{n}}$. The values of $\left(z^{\prime}, z^{\prime \prime}\right)$ can be obtained from Table 2.1 for a given, $z$, $n$. The values of $\alpha$ and $\beta$ are given by

$$
\begin{gathered}
\beta=\frac{F\left(z^{\prime \prime}\right)-F\left(z^{\prime}\right)}{z^{\prime \prime}-z^{\prime}} \\
\alpha=h_{2}\left(z^{\prime \prime}\right)-\beta z^{\prime \prime} \text { or } h_{2}\left(z^{\prime}\right)-\beta z^{\prime}
\end{gathered}
$$

The values of $\alpha, \beta$ for $n=3(1) 10$ and $r=1,2, \ldots, n-2$, were calculated and are presented in Table 3. With a specific choice of $n$ and $r$ the modified maximum likelihood estimate by this method is given by Equation (22), Let it be denoted by $\hat{\sigma_{1}}$. The bias, variance and mean square error of $\hat{\sigma_{1}}$ are calculated using the moments of standard gamma order statistics, given by Gupta (1960), Prescott (1974). These are given in Table 5.

\section{Method II:}

In this method Taylor's series expansion of $h_{2}(z)$ in the neighborhood of $(r+1)^{\text {th }}$ quantile of the population up to the first derivative is considered, so that $h_{2}\left(z_{\mathrm{r}+1}\right)$ is approximately equal to $\alpha+\beta z_{r+1}$ where

$$
\beta=h_{2}^{\prime}\left(\xi_{r+1}\right)
$$

Here $\xi_{r+1}$ is the solution of $F\left(\xi_{r+1}\right)=\frac{r+1}{n+1}$.

$$
\alpha=h_{2}\left(\xi_{r+1}\right)-\beta \xi_{r+1}
$$

Calculating $\xi_{r+1}$ by interpolation from Table 1 for $n=3(1) 10$ and $r=1,2, \ldots, n-2$, which in turn are used in Equations (25), (26) to obtain $\alpha$ and $\beta$. These values of $\alpha$ and $\beta$ can be used in Equation (22) to get another linear estimator as modified 
ESTIMATION FROM SAMPLES IN EXPONENTIAL \& GAMMA MODELS

maximum likelihood estimator say $\hat{\sigma_{2}}$. For the chosen combinations of $\mathrm{n}$ and $r$ the values of $\alpha$ and $\beta$ by this method are given in Table 3. As described in Method I the bias, variance and mean square error of $\hat{\sigma_{2}}$ are given in Table 5 .

\section{Method III:}

In this method the Taylor's series expansion of $h_{2}(z)$ in the neighborhood of expected value of $(r+1)^{\text {th }}$ standard order statistic, up to first derivative say $e_{r+1}$ i.e., $E\left(z_{r+1}\right)=e_{r+1}$ was considered. If the linear approximation is denoted by the same notation as that of Methods I and II without any scope for confusion results in

$$
h_{2}\left(z_{r+1}\right) \cong \alpha+\beta z_{r+1}
$$

where

$$
\beta=h_{2}^{\prime}\left(e_{r+1}\right)
$$

and

$$
\alpha=h_{2}\left(e_{r+1}\right)-\beta\left(e_{r+1}\right)
$$

Using the moments of gamma order statistics given by Gupta (1960) $\alpha$ and $\beta$ for $n=3(1) 10$ and $r=1,2, \ldots, n-2$ have been evaluated, these are given in Table 3 . Substituting the values of $\alpha$ and $\beta$ in Equation (22) results in another modified maximum likelihood estimator of $\hat{\sigma}_{3}$ for example,

$$
\sigma_{3}^{\wedge}=\sum_{i=r+1}^{n} 1_{i} x_{i}
$$

Where

$$
1_{i}=\frac{1-r \beta}{r \alpha+2(n-r)}, i=r+1
$$

$$
=\frac{1}{r \alpha+2(n-r)}, \quad i=r+2 \ldots \ldots ., n
$$

$\alpha, \beta$ in the above $1_{i}$ 's are given in Table 3 for a specific choice of $r$ and $n$. Because $\hat{\sigma_{3}}$ is also a linear estimator its bias, variance and mean square error can be calculated using the moments of gamma order statistics. These three sampling 


\section{KANTAM \& SRIRAM}

characteristics of $\hat{\sigma_{3}}$ are given in Table 5. For the sake of comparison of $\hat{\sigma_{1}} \hat{\sigma}_{2}, \hat{\sigma_{3}}$ which are linear estimators the variance of BLUE from a left censored sample of a gamma distribution borrowed from Kantam et al. (1989) is also included in Table 5.

As a matter of academic interest and comparison between CFR and a specific IFR model the calculations for exponential distribution have also been included. Table 6 gives the $\alpha, \beta$ values for $n$ and $r$. Table 7 gives the bias, variance, MSE of $\hat{\sigma_{1}} \hat{\sigma}_{2}, \hat{\sigma_{3}}$ respectively.

\section{Asymptotica Variance}

The log-likelihood equation to estimate $\hat{\sigma}$ from the left censored sample $\left(r_{1}=r, r_{2}\right.$ $=0)$ can be written as

$$
\frac{\partial \log L}{\partial \sigma}=\mathrm{h}_{2}(z)-2(\mathrm{n}-\mathrm{r})-\sum_{\mathrm{i}=\mathrm{r}+1}^{\mathrm{n}} \mathrm{z}_{\mathrm{i}}
$$

where $h_{2}(z)=\frac{\mathrm{z}^{2} e^{-z}}{1-e^{-z}(1+z)}$.

In the suggested three methods of modifications $h_{2}(z)$ as $\alpha+\beta z$ in the neighborhood of population's $r+1$ quantile or population's mean value of $(r+1)$ sample order statistics were approximated. Because sample quantiles are consistent estimators of population quantiles, sample moments are consistent estimators of population moments, in all the three methods of modification the function $h_{2}(z)$ is approximated as a linear function of $z$. For large values of $n$ the neighborhood of population quantile or population mean value becomes narrower thereby giving more linearity of $h_{2}(z)$ in that neighborhood, that is the closeness of $h_{2}(z)$ to $\alpha+\beta z$ is stronger, the larger the sample size. Hence for large $\mathrm{n}, \frac{\partial \log L}{\partial \sigma}$ is almost equal to $\alpha+\beta z$.

$$
\frac{\partial \log L}{\partial \sigma} \cong \frac{\partial \log L}{\partial \sigma}
$$

where 


$$
\begin{aligned}
& \frac{\partial \log L^{\prime}}{\partial \sigma}=\left(\alpha+\beta \mathrm{z}_{\mathrm{r}+1}\right)-2(n-r)-\sum_{i=r+1}^{n} z_{i} \\
& \therefore \quad E\left(\frac{\partial \log L}{\partial \sigma}\right)=E\left(\frac{\partial \log L^{\prime}}{\partial \sigma}\right) \text { for large } n \\
& E\left(\frac{\partial^{2} \log L}{\partial \sigma^{2}}\right)=E\left(\frac{\partial^{2} \log L^{\prime}}{\partial \sigma^{2}}\right) \text { for large } n
\end{aligned}
$$

MMLEs of Method I, Method II, Method III have the same asymptotic bias, asymptotic variance as that of MLE. Also BLUE of $\sigma$ is asymptotically as efficient as MLE and conversely MLEs are asymptotically linear (David 1981, p. 136., p. 273). As such the three MMLEs are asymptotically equivalent to BLUE, exact MLE in the case of left censored samples. For a right censored sample it is the exact MLE and BLUE that stand in competition, which are asymptotically equal.

\section{Small Sample Comparison}

The following comparative conclusions from Table 4 are drawn about the performance of exact MLE, BLUE in right censored sample. With respect to variance as well as MSE, it is maximum likelihood estimator that is preferable to BLUE from right censored samples. In case of left censored samples, from Table 5 MMLE under Method I is the recommended estimator.for exponential population also the MMLE under Method I is most preferable.

\section{Reliability Estimation}

The reliability of any item whose life time variate follows gamma distribution is given by

$$
R(x)=e^{-\frac{x}{\sigma}}\left(1+\frac{\mathrm{x}}{\sigma}\right)
$$

where $\sigma$ a scale parameter and $x$ is is the time instant at which the reliability is desired. If $\sigma$ is not known reliability is to be estimated through estimators of $\sigma$ or otherwise Next an attempt is made to estimate reliability using the BLUE, MLE, MMLEs of three methods of Section 2. It is proposed that the corresponding 


\section{KANTAM \& SRIRAM}

estimate of reliability with $\sigma$ in $R(x)$ replaced by $\hat{\sigma}^{\wedge}$. This is analogous to the wellknown invariance property of ML method of estimation. Though the non ML methods of estimation in this discussion are not established to have invariance property it is exploited here as per remark in Sinha (1986). Accordingly four additional estimators of reliability corresponding to the four methods of estimation of $\sigma$ are obtained. For one such estimator of reliability the asymptotic variance is given by

$$
\operatorname{as} \operatorname{var} \hat{R}(\mathrm{x} ; \sigma)=\left(\frac{\partial \mathrm{R}}{\partial \sigma}\right)^{2} \cdot \operatorname{asvar}(\hat{\sigma})
$$

where as $\operatorname{var}(\hat{\sigma})$ is the asymptotic variance of $\hat{\sigma}$ obtained by a particular method. For a gamma distribution with shape 2 and scale parameter $\sigma$, Equation (31) becomes

$$
\operatorname{as} \operatorname{var} \hat{R}(z)=\frac{\mathrm{z}^{4} \mathrm{e}^{-2 \mathrm{z}}}{\sigma^{2}} \cdot \operatorname{asvar}(\hat{\sigma})
$$

where $z=x / \sigma$.

Therefore all the five reliability estimates are asymptotically equally efficient as explained in Section 2. At the same time the small sample variances of the reliability estimates are not mathematically tractable. Monte-Carlo simulation method was used to assess the small sample behavior. 3,000 samples of size $n=$ 3(1)10 are generated from a gamma distribution. For each sample after ordering all possible right censored situation with $r=1(1) n-2$ are extracted. For each right censored sample the exact MLE as the unique positive root of quadratic Equation (11) is computed say $\hat{\sigma_{M}}$. In order to get the BLUE of $\sigma$ for the right censored sample the coefficients of uncensored observations for a gamma distribution need to be supplied by a general formula of Lloyd (1952) in scaled densities. As these coefficients are not exhaustively available in published form, they have been evaluated making use of the expected values of Gupta (1960) and variance covariances of Prescott (1974) of gamma order statistics. These coefficients are used to get the BLUE of $\sigma$ from a right censored sample say $\hat{\sigma}_{B}$. The gamma reliability function $R(x ; \sigma)$ is calculated with $\sigma$ replaced by $\hat{\sigma^{\wedge}}, \hat{\sigma}_{B}$ in succession at values of $x$ corresponding to $R(x)=0.1(0.1) 0.9$. The bias, the variance and MSE of the estimated reliabilities for a given $x, n, r$ a method of estimation across the 


\section{ESTIMATION FROM SAMPLES IN EXPONENTIAL \& GAMMA MODELS}

3,000 simulated runs are evaluated and these empirical sample characteristic are presented in Table 8.

For left censored samples the exact MLE $\hat{\sigma}_{M}$ for each simulated sample is obtained as an iterative solution of Equation (15) by Newton-raphson method. The BLUE $\hat{\sigma}_{B}$ is computed as narrated in the case of right censored samples with the distinction that the weights are calculated for the suffixes of available observations. The three modified maximum likelihood estimators $\hat{\sigma}_{1}, \hat{\sigma}_{2}, \hat{\sigma}_{3}$ of the Methods I, II, III respectively are also calculated for each simulated sample using the constants of linearization given in Table 3 in Equation (22).The reliability estimate of left censored sample is obtained in five different ways by successively replacing $\sigma$ in $R(x ; \sigma)$ with $\hat{\sigma}_{M}, \hat{\sigma}_{B}, \hat{\sigma}_{1}, \hat{\sigma}_{2}, \hat{\sigma}_{3}$ at values of $x$ corresponding to $R(x)=0.1(0.1) 0.9$ for all left censored samples with $\mathrm{n}=3(1) 10, r=1(1) n-2$. The empirical bias, variance, MSE of all the reliability estimates across the 3,000 simulation runs are presented in Table 9 for $R(x)=0.9$ only a byproduct this entire work is carried out for exponential distribution also in the case of left censored sample only. These results are given in Table 10 .

The competition of rating for preferability fell between the use of MLE/BLUE to estimate the gamma reliability function from right censored samples. In the case of left censored samples Method I or Method II of MMLE are therefore suggested. In the case of single parameter exponential distribution the use of BLUE is suggested, although the use of MML Method I or the MML Method II are equally good.

\section{References}

Balakrishnan. N., \& Cohen, A. C. (1990). Order statistics and inference. London: Academic Press.

Balasooriya, U., \& Hapuaracchihi (1992). Extended tables for the moments of gamma distribution order statistics. IEEE Transactions on Reliability, 41(2).

Cohen, A. C., \& Whitten, B. (1982). Modified moment and maximum likelihood estimators for parameters of the three-parameter gamma distribution. Commun. Statist.-Simu. Comp., 11, 197-216.

David, H. A. (1981). Order statistics. New York: John Wiley and Sons.

Gupta, S. S. (1960). Order statistics from the gamma distribution. Technometrics, 2, 243-262. 


\section{KANT AM \& SRIRAM}

Kantam, R. R. L., Anjaneyulu, G. V. S. R., \& Narasimham, V.L. (1989). On linear estimation of gamma scale parameter from censored samples. Assam Statist.Rev., 3(1), 1-21.

Kantam, R. R. L., \& Srinivasa, R. G. (2002). Log-Logistic distribution: Modified Maximum Likelihood estimation. Gujarat Statistical Review, 29(1-2), 25-36.

Kantam, R. R. L., Srinivasa, R. G., \& Sriram,B. (2007). On stress-strength reliability model in log-logistic distribution. International Journal of Agricultural Statistical Science, 3(1), 1-6.

Lloyd, E. H. (1952). Least-squares estimation of location and scale parameters using order statistics. Biometrika, 39, 88-95.

Mehrotra, K. G., \& Nanda, P. (1974). Unbiased estimation of parameters by order statistics in the case of censored samples. Biometrika, 61, 601-606.

Prescott, P. (1974). Variances and covariances of order statistics from the gamma distribution. Biometrika, 61, 607-613.

Rosaiah, K., Kamtam, R. R. L. \& Narasimham, V. L. (1993a). ML and modified ML estimation in gamma distribution with a known prior relation among the parameters. Pakistan Journal of Statistics, 9(3), B, 37-48.

Rosaiah, K. Kamtam, R. R. L., \& Narasimham, V. L. (1993b). On modified maximum likelihood estimation of gamma parameters. Journal of Statistical Research, Bangladesh, 27(1-2), 15-28.

Rosaiah, K., Kantam, R. R. L., \& Jayaprakash, B. (1997). Reliability estimation in half logistic model. Gujarat Statistical Review, 24(2), 11-18.

Rosaiah, K., Kantam, R. R. L., \& Pratapa Reddy, J. (2007). Estimation in inverse Rayleigh distribution. International Journal of Agricultural Statistical Science, 3(2), 453-470.

Sinha, S. K. (1986). Reliability and life testing. Wiley Eastern Limited.

Srinivasa, R. G. \& Kantam, R. R. L. (2004). A note on point estimation of system reliability exemplified for the log-logistic distribution. Economic Quality Control, 19(2), 197-204.

Tiku, M. L. (1967). Estimating the mean and standard deviation from a censored normal sample. Biometrika, 54, 155-165.

Tiku, M. L. \& Suresh, R. P. (1992). A new method of estimation for location and scale parameters. Journal of Statistical Planning and Inference, 30, 281-292. 


\section{ESTIMATION FROM SAMPLES IN EXPONENTIAL \& GAMMA MODELS}

\section{Appendix A}

Table 1. Values of cumulative distribution function of Gamma distribution with shape parameter 2

\begin{tabular}{|c|c|c|c|c|c|c|c|c|c|c|}
\hline$Z$ & 0 & 0.01 & 0.02 & 0.03 & 0.04 & 0.05 & 0.06 & 0.07 & 0.08 & 0.09 \\
\hline 0.00 & 0.00000 & 0.00005 & 0.00019 & 0.00044 & 0.00077 & 0.00120 & 0.00173 & 0.00233 & 0.00303 & 0.00381 \\
\hline 0.10 & 0.00467 & 0.00562 & 0.00664 & 0.00775 & 0.00893 & 0.01018 & 0.01151 & 0.01291 & 0.01438 & 0.01591 \\
\hline 0.20 & 0.01752 & 0.01919 & 0.02092 & 0.02272 & 0.02458 & 0.02649 & 0.02847 & 0.03050 & 0.03259 & 0.03474 \\
\hline 0.30 & 0.03693 & 0.03918 & 0.04148 & 0.04383 & 0.04622 & 0.04867 & 0.05116 & 0.05369 & 0.05627 & 0.05889 \\
\hline 0.40 & 0.06155 & 0.06425 & 0.06699 & 0.06977 & 0.07258 & 0.07543 & 0.07832 & 0.08124 & 0.08420 & 0.08918 \\
\hline 0.50 & 0.09020 & 0.09325 & 0.09632 & 0.09943 & 0.10256 & 0.10572 & 0.10891 & 0.11212 & 0.11536 & 0.11862 \\
\hline 0.60 & 0.12190 & 0.12520 & 0.12853 & 0.13187 & 0.13524 & 0.13862 & 0.14202 & 0.14544 & 0.14888 & 0.15233 \\
\hline 0.70 & 0.15580 & 0.15928 & 0.16278 & 0.16629 & 0.16982 & 0.17335 & 0.17690 & 0.18046 & 0.18403 & 0.18761 \\
\hline 0.80 & 0.19120 & 0.19480 & 0.19841 & 0.20203 & 0.20565 & 0.20928 & 0.21291 & 0.21656 & 0.22020 & 0.22386 \\
\hline 0.90 & 0.22751 & 0.23117 & 0.23484 & 0.23851 & 0.24218 & 0.24585 & 0.24953 & 0.25320 & 0.25688 & 0.26056 \\
\hline 1.00 & 0.26424 & 0.26792 & 0.27159 & 0.27527 & 0.27895 & 0.28262 & 0.28630 & 0.28997 & 0.29364 & 0.29730 \\
\hline 1.10 & 0.30097 & 0.30463 & 0.30828 & 0.31193 & 0.31558 & 0.31923 & 0.32287 & 0.32650 & 0.33013 & 0.33375 \\
\hline 1.20 & 0.33737 & 0.34098 & 0.34458 & 0.34818 & 0.35177 & 0.35536 & 0.35894 & 0.36251 & 0.36607 & 0.36963 \\
\hline 1.30 & 0.37317 & 0.37671 & 0.38024 & 0.38376 & 0.38728 & 0.39078 & 0.39428 & 0.39776 & 0.40124 & 0.40471 \\
\hline 1.40 & 0.40816 & 0.41161 & 0.41505 & 0.41847 & 0.42189 & 0.42530 & 0.42869 & 0.43208 & 0.43545 & 0.43882 \\
\hline 1.50 & 0.44217 & 0.44551 & 0.44884 & 0.45216 & 0.45547 & 0.45876 & 0.46205 & 0.46532 & 0.46858 & 0.47183 \\
\hline 1.60 & 0.47506 & 0.47829 & 0.48150 & 0.48470 & 0.48789 & 0.49106 & 0.49423 & 0.49738 & 0.50051 & 0.50364 \\
\hline 1.70 & 0.50675 & 0.50985 & 0.51294 & 0.51601 & 0.51907 & 0.52212 & 0.52515 & 0.52817 & 0.53118 & 0.53418 \\
\hline 1.80 & 0.53716 & 0.54013 & 0.54308 & 0.54603 & 0.54895 & 0.55187 & 0.55477 & 0.55766 & 0.56054 & 0.56340 \\
\hline 1.90 & 0.56625 & 0.56908 & 0.57190 & 0.57471 & 0.57751 & 0.58029 & 0.83059 & 0.58581 & 0.58855 & 0.59128 \\
\hline 2.00 & 0.59399 & 0.59669 & 0.59938 & 0.60205 & 0.60471 & 0.60735 & 0.60999 & 0.61261 & 0.61521 & 0.61780 \\
\hline 2.10 & 0.62038 & 0.62295 & 0.62550 & 0.62803 & 0.63056 & 0.63307 & 0.63557 & 0.63805 & 0.64052 & 0.64298 \\
\hline 2.20 & 0.64543 & 0.64786 & 0.65027 & 0.65268 & 0.65507 & 0.65745 & 0.65981 & 0.66216 & 0.66450 & 0.66683 \\
\hline 2.30 & 0.66914 & 0.67144 & 0.67373 & 0.67600 & 0.67826 & 0.68051 & 0.68274 & 0.68497 & 0.68717 & 0.68937 \\
\hline 2.40 & 0.69155 & 0.69373 & 0.69588 & 0.69803 & 0.70016 & 0.70228 & 0.70439 & 0.70649 & 0.70857 & 0.71064 \\
\hline 2.50 & 0.71270 & 0.71474 & 0.71675 & 0.71880 & 0.72081 & 0.72281 & 0.72479 & 0.72676 & 0.72872 & 0.73067 \\
\hline 2.60 & 0.73261 & 0.73454 & 0.73645 & 0.73835 & 0.74024 & 0.74212 & 0.74399 & 0.74584 & 0.74768 & 0.74951 \\
\hline 2.70 & 0.75134 & 0.75314 & 0.75494 & 0.75673 & 0.75850 & 0.76027 & 0.76202 & 0.76376 & 0.76549 & 0.76721 \\
\hline 2.80 & 0.76892 & 0.77061 & 0.77230 & 0.77398 & 0.77564 & 0.77729 & 0.77894 & 0.78057 & 0.78219 & 0.78380 \\
\hline 2.90 & 0.78540 & 0.78700 & 0.78858 & 0.79015 & 0.79170 & 0.79325 & 0.79479 & 0.79632 & 0.79784 & 0.79935 \\
\hline
\end{tabular}




\section{KANTAM \& SRIRAM}

Table 1 (contd.). Values of cumulative distribution function of Gamma distribution with shape parameter 2

\begin{tabular}{|c|c|c|c|c|c|c|c|c|c|c|}
\hline$Z$ & 0 & 0.01 & 0.02 & 0.03 & 0.04 & 0.05 & 0.06 & 0.07 & 0.08 & 0.09 \\
\hline 3.00 & 0.80085 & 0.80234 & 0.80381 & 0.80528 & 0.80674 & 0.80819 & 0.80963 & 0.81106 & 0.81248 & 0.81389 \\
\hline 3.10 & 0.81529 & 0.81669 & 0.81807 & 0.81944 & 0.82080 & 0.82216 & 0.82350 & 0.82484 & 0.82617 & 0.82749 \\
\hline 3.20 & 0.82879 & 0.83009 & 0.83139 & 0.83267 & 0.83394 & 0.83521 & 0.83646 & 0.83771 & 0.83895 & 0.84018 \\
\hline 3.30 & 0.84140 & 0.84261 & 0.84382 & 0.84501 & 0.84620 & 0.84738 & 0.84855 & 0.84971 & 0.85087 & 0.85201 \\
\hline 3.40 & 0.85315 & 0.85418 & 0.85541 & 0.85652 & 0.85763 & 0.85873 & 0.85982 & 0.86090 & 0.86198 & 0.86305 \\
\hline 3.50 & 0.86411 & 0.86516 & 0.86621 & 0.86724 & 0.86827 & 0.86930 & 0.87031 & 0.87132 & 0.87232 & 0.87332 \\
\hline 3.60 & 0.87431 & 0.87529 & 0.87626 & 0.87723 & 0.87818 & 0.87914 & 0.88008 & 0.88102 & 0.88195 & 0.88288 \\
\hline 3.70 & 0.88379 & 0.88471 & 0.88561 & 0.88651 & 0.88740 & 0.88829 & 0.88916 & 0.89004 & 0.89090 & 0.89176 \\
\hline 3.80 & 0.89262 & 0.89346 & 0.89430 & 0.89514 & 0.89597 & 0.89679 & 0.89761 & 0.89842 & 0.89922 & 0.90002 \\
\hline 3.90 & 0.90082 & 0.90160 & 0.90238 & 0.90316 & 0.90393 & 0.90469 & 0.90545 & 0.90620 & 0.90695 & 0.90769 \\
\hline 4.00 & 0.90842 & 0.90915 & 0.90988 & 0.91060 & 0.91131 & 0.91202 & 0.91272 & 0.91342 & 0.91411 & 0.91480 \\
\hline 4.10 & 0.91548 & 0.91616 & 0.91683 & 0.91750 & 0.91816 & 0.91881 & 0.91947 & 0.92011 & 0.92075 & 0.92139 \\
\hline 4.20 & 0.92202 & 0.92265 & 0.92327 & 0.92389 & 0.92451 & 0.92511 & 0.92572 & 0.92632 & 0.92691 & 0.92750 \\
\hline 4.30 & 0.92809 & 0.92867 & 0.92925 & 0.92982 & 0.93039 & 0.93095 & 0.93151 & 0.93206 & 0.93261 & 0.93316 \\
\hline 4.40 & 0.93370 & 0.93424 & 0.93478 & 0.93531 & 0.93583 & 0.93635 & 0.93687 & 0.93738 & 0.93789 & 0.93840 \\
\hline 4.50 & 0.93890 & 0.93940 & 0.93989 & 0.94038 & 0.94087 & 0.94135 & 0.94183 & 0.94231 & 0.94278 & 0.94325 \\
\hline 4.60 & 0.94371 & 0.94417 & 0.94463 & 0.94508 & 0.94553 & 0.94598 & 0.94642 & 0.94686 & 0.94730 & 0.94773 \\
\hline 4.70 & 0.94816 & 0.94858 & 0.94901 & 0.94943 & 0.94984 & 0.95025 & 0.95066 & 0.95107 & 0.95147 & 0.95187 \\
\hline 4.80 & 0.95227 & 0.95266 & 0.95305 & 0.95344 & 0.95382 & 0.95420 & 0.95458 & 0.95496 & 0.95533 & 0.95570 \\
\hline 4.90 & 0.95607 & 0.95643 & 0.95679 & 0.95715 & 0.95750 & 0.95785 & 0.95820 & 0.95855 & 0.95889 & 0.95923 \\
\hline 5.00 & 0.95957 & 0.95991 & 0.96024 & 0.96057 & 0.96090 & 0.96122 & 0.96155 & 0.96187 & 0.96218 & 0.96250 \\
\hline 5.10 & 0.96281 & 0.96312 & 0.96343 & 0.96373 & 0.96403 & 0.96433 & 0.96463 & 0.96493 & 0.96522 & 0.96551 \\
\hline 5.20 & 0.96580 & 0.96608 & 0.96637 & 0.96665 & 0.96693 & 0.96720 & 0.96748 & 0.96775 & 0.96802 & 0.96829 \\
\hline 5.30 & 0.96855 & 0.96882 & 0.96908 & 0.96934 & 0.96959 & 0.96985 & 0.97010 & 0.97035 & 0.97060 & 0.97085 \\
\hline 5.40 & 0.97109 & 0.97134 & 0.97158 & 0.97182 & 0.97205 & 0.97229 & 0.97252 & 0.97275 & 0.97298 & 0.97321 \\
\hline 5.50 & 0.97344 & 0.97366 & 0.97388 & 0.97410 & 0.97432 & 0.97454 & 0.97475 & 0.97497 & 0.97518 & 0.97539 \\
\hline 5.60 & 0.97559 & 0.97580 & 0.97601 & 0.97621 & 0.97641 & 0.97661 & 0.97681 & 0.97700 & 0.97720 & 0.97739 \\
\hline 5.70 & 0.97758 & 0.97777 & 0.97796 & 0.97815 & 0.97833 & 0.97852 & 0.97870 & 0.97888 & 0.97906 & 0.97924 \\
\hline 5.80 & 0.97941 & 0.97959 & 0.97976 & 0.97993 & 0.98010 & 0.98027 & 0.98044 & 0.98061 & 0.98077 & 0.98094 \\
\hline 5.90 & 0.98110 & 0.98126 & 0.98142 & 0.98158 & 0.98173 & 0.98189 & 0.98204 & 0.98220 & 0.98235 & 0.98250 \\
\hline
\end{tabular}




\section{ESTIMATION FROM SAMPLES IN EXPONENTIAL \& GAMMA MODELS}

Table 1 (contd.). Values of cumulative distribution function of Gamma distribution with shape parameter 2

\begin{tabular}{|c|c|c|c|c|c|c|c|c|c|c|}
\hline$Z$ & 0 & 0.01 & 0.02 & 0.03 & 0.04 & 0.05 & 0.06 & 0.07 & 0.08 & 0.09 \\
\hline 6.00 & 0.98265 & 0.98280 & 0.98294 & 0.98309 & 0.98323 & 0.98338 & 0.98352 & 0.98366 & 0.98380 & 0.98394 \\
\hline 6.10 & 0.98408 & 0.98421 & 0.98435 & 0.98448 & 0.98461 & 0.98475 & 0.98488 & 0.98501 & 0.98513 & 0.98526 \\
\hline 6.20 & 0.98539 & 0.98551 & 0.98564 & 0.98576 & 0.98588 & 0.98600 & 0.98612 & 0.98624 & 0.98636 & 0.98648 \\
\hline 6.30 & 0.98660 & 0.98671 & 0.98682 & 0.98694 & 0.98705 & 0.98716 & 0.98727 & 0.98738 & 0.98749 & 0.98760 \\
\hline 6.40 & 0.98771 & 0.98781 & 0.98792 & 0.98802 & 0.98812 & 0.98823 & 0.98833 & 0.98843 & 0.98852 & 0.98832 \\
\hline 6.50 & 0.98872 & 0.98882 & 0.98892 & 0.98901 & 0.98911 & 0.98920 & 0.98930 & 0.98939 & 0.98948 & 0.98957 \\
\hline 6.60 & 0.98966 & 0.98975 & 0.98984 & 0.98993 & 0.99001 & 0.99010 & 0.99019 & 0.99027 & 0.99036 & 0.99044 \\
\hline 6.70 & 0.99052 & 0.99060 & 0.99069 & 0.99077 & 0.99085 & 0.99093 & 0.99100 & 0.99108 & 0.99116 & 0.99124 \\
\hline 6.80 & 0.99131 & 0.99139 & 0.99146 & 0.99154 & 0.99161 & 0.99168 & 0.99176 & 0.99183 & 0.99190 & 0.99197 \\
\hline 6.90 & 0.99204 & 0.99211 & 0.99218 & 0.99224 & 0.99231 & 0.99238 & 0.99245 & 0.99251 & 0.99258 & 0.99264 \\
\hline 7.00 & 0.99271 & 0.99277 & 0.99283 & 0.99289 & 0.99296 & 0.99302 & 0.99308 & 0.99314 & 0.99320 & 0.99326 \\
\hline 7.10 & 0.99332 & 0.99338 & 0.99343 & 0.99349 & 0.99355 & 0.99360 & 0.99366 & 0.99372 & 0.99377 & 0.99382 \\
\hline 7.20 & 0.99388 & 0.99393 & 0.99399 & 0.99404 & 0.99409 & 0.99414 & 0.99419 & 0.99424 & 0.99429 & 0.99434 \\
\hline 7.30 & 0.99439 & 0.99444 & 0.99449 & 0.99454 & 0.99459 & 0.99463 & 0.99468 & 0.99473 & 0.99477 & 0.99482 \\
\hline 7.40 & 0.99487 & 0.99491 & 0.99496 & 0.99500 & 0.99504 & 0.99509 & 0.99513 & 0.99517 & 0.99522 & 0.99526 \\
\hline 7.50 & 0.99530 & 0.99534 & 0.99538 & 0.99542 & 0.99546 & 0.99550 & 0.99554 & 0.99558 & 0.99562 & 0.99566 \\
\hline 7.60 & 0.99570 & 0.99573 & 0.99577 & 0.99581 & 0.99585 & 0.99588 & 0.99592 & 0.99595 & 0.99599 & 0.99603 \\
\hline 7.70 & 0.99606 & 0.99610 & 0.99613 & 0.99616 & 0.99620 & 0.99623 & 0.99626 & 0.99630 & 0.99633 & 0.99636 \\
\hline 7.80 & 0.99639 & 0.99643 & 0.99646 & 0.99649 & 0.99652 & 0.99655 & 0.99658 & 0.99661 & 0.99664 & 0.99667 \\
\hline 7.90 & 0.99670 & 0.99643 & 0.99676 & 0.99679 & 0.99682 & 0.99684 & 0.99687 & 0.99690 & 0.99693 & 0.99695 \\
\hline 8.00 & 0.99698 & 0.99701 & 0.99703 & 0.99706 & 0.99709 & 0.99711 & 0.99714 & 0.99716 & 0.99719 & 0.99721 \\
\hline 8.10 & 0.99724 & 0.99726 & 0.99729 & 0.99731 & 0.99733 & 0.99736 & 0.99738 & 0.99741 & 0.99743 & 0.99745 \\
\hline 8.20 & 0.99747 & 0.99750 & 0.99752 & 0.99754 & 0.99756 & 0.99758 & 0.99761 & 0.99763 & 0.99765 & 0.99767 \\
\hline 8.30 & 0.99769 & 0.99771 & 0.99773 & 0.99775 & 0.99777 & 0.99779 & 0.99781 & 0.99783 & 0.99779 & 0.99787 \\
\hline 8.40 & 0.99789 & 0.99791 & 0.99792 & 0.99794 & 0.99796 & 0.99798 & 0.99800 & 0.99801 & 0.99803 & 0.99805 \\
\hline 8.50 & 0.99807 & 0.99808 & 0.99810 & 0.99812 & 0.99814 & 0.99815 & 0.99817 & 0.99818 & 0.99820 & 0.99822 \\
\hline 8.60 & 0.99823 & 0.99825 & 0.99826 & 0.99828 & 0.99830 & 0.99831 & 0.99833 & 0.99834 & 0.99836 & 0.99837 \\
\hline 8.70 & 0.99838 & 0.99840 & 0.99841 & 0.99843 & 0.99844 & 0.99846 & 0.99847 & 0.99848 & 0.99850 & 0.99851 \\
\hline 8.80 & 0.99852 & 0.99854 & 0.99855 & 0.99856 & 0.99858 & 0.99859 & 0.99860 & 0.99861 & 0.99863 & 0.99864 \\
\hline 8.90 & 0.99865 & 0.99866 & 0.99867 & 0.99869 & 0.99870 & 0.99871 & 0.99872 & 0.99873 & 0.99874 & 0.99876 \\
\hline
\end{tabular}




\section{KANTAM \& SRIRAM}

Table 1 (contd.). Values of cumulative distribution function of Gamma distribution with shape parameter 2

\begin{tabular}{|c|c|c|c|c|c|c|c|c|c|c|}
\hline$Z$ & 0 & 0.01 & 0.02 & 0.03 & 0.04 & 0.05 & 0.06 & 0.07 & 0.08 & 0.09 \\
\hline 9.00 & 0.99877 & 0.99878 & 0.99879 & 0.99880 & 0.99881 & 0.99882 & 0.99883 & 0.99884 & 0.99885 & 0.99886 \\
\hline 9.10 & 0.99887 & 0.99888 & 0.99889 & 0.99890 & 0.99891 & 0.99892 & 0.99893 & 0.99894 & 0.99895 & 0.99896 \\
\hline 9.20 & 0.99897 & 0.99898 & 0.99899 & 0.99900 & 0.99901 & 0.99902 & 0.99902 & 0.99903 & 0.99904 & 0.99905 \\
\hline 9.30 & 0.99906 & 0.99907 & 0.99908 & 0.99908 & 0.99909 & 0.99910 & 0.99911 & 0.99912 & 0.99912 & 0.99913 \\
\hline 9.40 & 0.99914 & 0.99915 & 0.99916 & 0.99916 & 0.99917 & 0.99918 & 0.99919 & 0.99919 & 0.99920 & 0.99921 \\
\hline 9.50 & 0.99924 & 0.99922 & 0.99923 & 0.99924 & 0.99924 & 0.99925 & 0.99926 & 0.99926 & 0.99927 & 0.99928 \\
\hline 9.60 & 0.99928 & 0.99929 & 0.99930 & 0.99930 & 0.99931 & 0.99931 & 0.99932 & 0.99933 & 0.99933 & 0.99934 \\
\hline 9.70 & 0.99934 & 0.99935 & 0.99936 & 0.99936 & 0.99937 & 0.99937 & 0.99938 & 0.99939 & 0.99939 & 0.99940 \\
\hline 9.80 & 0.99401 & 0.99941 & 0.99941 & 0.99942 & 0.99942 & 0.99943 & 0.99943 & 0.99944 & 0.99944 & 0.99945 \\
\hline 9.90 & 0.99945 & 0.99946 & 0.99946 & 0.99947 & 0.99948 & 0.99948 & 0.99948 & 0.99949 & 0.99949 & 0.99950 \\
\hline 10.00 & 0.99950 & 0.99951 & 0.99951 & 0.99951 & 0.99952 & 0.99952 & 0.99953 & 0.99953 & 0.99954 & 0.99954 \\
\hline 10.10 & 0.99954 & 0.99955 & 0.99955 & 0.99956 & 0.99956 & 0.99956 & 0.99957 & 0.99957 & 0.99958 & 0.99958 \\
\hline 10.20 & 0.99958 & 0.99959 & 0.99959 & 0.99960 & 0.99960 & 0.99960 & 0.99961 & 0.99961 & 0.99961 & 0.99962 \\
\hline 10.30 & 0.99962 & 0.99962 & 0.99963 & 0.99963 & 0.99963 & 0.99964 & 0.99964 & 0.99964 & 0.99965 & 0.99965 \\
\hline 10.40 & 0.99965 & 0.99966 & 0.99966 & 0.99966 & 0.99967 & 0.99967 & 0.99967 & 0.99968 & 0.99968 & 0.99968 \\
\hline 10.50 & 0.99968 & 0.99969 & 0.99969 & 0.99969 & 0.99970 & 0.99972 & 0.99970 & 0.99970 & 0.99971 & 0.99971 \\
\hline 10.60 & 0.99971 & 0.99971 & 0.99972 & 0.99972 & 0.99972 & 0.99975 & 0.99973 & 0.99973 & 0.99973 & 0.99973 \\
\hline 10.70 & 0.99974 & 0.99974 & 0.99974 & 0.99974 & 0.99975 & 0.99977 & 0.99975 & 0.99975 & 0.99976 & 0.99976 \\
\hline 10.80 & 0.99976 & 0.99976 & 0.99976 & 0.99977 & 0.99977 & 0.99979 & 0.99977 & 0.99977 & 0.99978 & 0.99978 \\
\hline 10.90 & 0.99978 & 0.99978 & 0.99978 & 0.99979 & 0.99979 & 0.99981 & 0.99979 & 0.99979 & 0.99980 & 0.99980 \\
\hline 11.00 & 0.99980 & 0.99980 & 0.99980 & 0.99981 & 0.99981 & 0.99983 & 0.99981 & 0.99981 & 0.99981 & 0.99982 \\
\hline 11.10 & 0.99982 & 0.99982 & 0.99982 & 0.99982 & 0.99982 & 0.99984 & 0.99983 & 0.99983 & 0.99983 & 0.99983 \\
\hline 11.20 & 0.99983 & 0.99984 & 0.99984 & 0.99984 & 0.99984 & 0.99986 & 0.99984 & 0.99984 & 0.99985 & 0.99985 \\
\hline 11.30 & 0.99985 & 0.99985 & 0.99985 & 0.99985 & 0.99985 & 0.99987 & 0.99986 & 0.99986 & 0.99986 & 0.99986 \\
\hline 11.40 & 0.99986 & 0.99986 & 0.99986 & 0.99987 & 0.99987 & 0.99987 & 0.99987 & 0.99987 & 0.99987 & 0.99987 \\
\hline 11.50 & 0.99987 & 0.99988 & 0.99988 & 0.99988 & 0.99988 & 0.99988 & 0.99988 & 0.99988 & 0.99988 & 0.99988 \\
\hline 11.60 & 0.99988 & 0.99988 & 0.99989 & 0.99989 & 0.99989 & 0.99989 & 0.99989 & 0.99989 & 0.99989 & 0.99989 \\
\hline 11.70 & 0.99990 & 0.99989 & 0.99990 & 0.99990 & 0.99990 & 0.99989 & 0.99990 & 0.99990 & 0.99990 & 0.99990 \\
\hline 11.80 & 0.99990 & 0.99991 & 0.99991 & 0.99991 & 0.99991 & 0.99991 & 0.99991 & 0.99991 & 0.99991 & 0.99991 \\
\hline
\end{tabular}




\section{ESTIMATION FROM SAMPLES IN EXPONENTIAL \& GAMMA MODELS}

Table 2. Gamma (2) distribution correlation coefficients of $\left(z, h_{1}(z)\right),\left(z, h_{2}(z)\right), z \in$ $\left(z^{*}, z^{* *}\right)$

\begin{tabular}{l|l|cccc}
\hline \multicolumn{1}{l}{$\boldsymbol{n}$} & $\boldsymbol{r}$ & $\boldsymbol{z}^{\boldsymbol{*}}$ & $\boldsymbol{z}^{* *}$ & $\boldsymbol{r}_{\mathbf{1}}$ & $\boldsymbol{r}_{\mathbf{2}}$ \\
\hline 5 & 1 & 0.6 & 1.83 & 0.9528 & 0.999 \\
& 2 & 1.03 & 2.56 & 0.9633 & 0.99838 \\
& 3 & 1.54 & 3.64 & 0.9626 & 0.99548 \\
\hline \multirow{3}{*}{0} & 1 & 0.39 & 1.11 & 0.94753 & 0.99177 \\
& 2 & 0.63 & 1.42 & 0.97025 & 0.9992 \\
& 3 & 0.86 & 1.73 & 0.97219 & 0.99897 \\
& 4 & 1.09 & 2.07 & 0.97798 & 0.99922 \\
& 5 & 1.34 & 2.45 & 0.979 & 0.9992 \\
& 6 & 1.63 & 2.92 & 0.97784 & 0.99693 \\
& 7 & 1.97 & 3.54 & 0.9769 & 0.99597 \\
& 8 & 2.42 & 4.53 & 0.9555 & 0.98629 \\
\hline
\end{tabular}




\section{KANTAM \& SRIRAM}

Table 3. Gamma (2) distribution constants of linear approximation for MMLEs from left censored sample

\begin{tabular}{|c|c|c|c|c|c|c|c|}
\hline \multirow[b]{2}{*}{$n$} & \multirow[b]{2}{*}{$r$} & \multicolumn{2}{|c|}{ Method I } & \multicolumn{2}{|c|}{ Method II } & \multicolumn{2}{|c|}{ Method III } \\
\hline & & $a$ & $b$ & $a$ & $b$ & $a$ & $b$ \\
\hline 3 & 1 & 1.84091 & -0.43072 & 1.81952 & -0.45748 & 1.78597 & -0.43832 \\
\hline \multirow{2}{*}{4} & 1 & 1.90320 & -0.48750 & 1.88015 & -0.49717 & 1.86883 & -0.48912 \\
\hline & 2 & 1.73474 & -0.37991 & 1.73608 & -0.41238 & 1.68453 & -0.38802 \\
\hline \multirow{3}{*}{5} & 1 & 1.93150 & -0.51815 & 1.91158 & -0.52169 & 1.90748 & -0.51826 \\
\hline & 2 & 1.83181 & -0.44270 & 1.81953 & -0.45749 & 1.79827 & -0.44516 \\
\hline & 3 & 1.64480 & -0.34330 & 1.66186 & -0.37794 & 1.59699 & -0.35086 \\
\hline \multirow{4}{*}{6} & 1 & 1.94746 & -0.53785 & 1.93053 & -0.53854 & 1.92936 & -0.56374 \\
\hline & 2 & 1.87819 & -0.47836 & 1.86467 & -0.48625 & 1.85453 & -0.47943 \\
\hline & 3 & 1.83834 & -0.43395 & 1.76285 & -0.42596 & 1.73350 & -0.41111 \\
\hline & 4 & 1.56715 & -0.31499 & 1.59551 & -0.35027 & 1.52114 & -0.32191 \\
\hline \multirow{5}{*}{7} & 1 & 1.95763 & -0.55182 & 1.94311 & -0.55099 & 1.94331 & -0.55119 \\
\hline & 2 & 1.90588 & -0.50233 & 1.89266 & -0.50650 & 1.88752 & -0.50261 \\
\hline & 3 & 1.82782 & -0.44717 & 1.81952 & -0.45748 & 1.80406 & -0.44845 \\
\hline & 4 & 1.70687 & -0.38095 & 1.71033 & -0.39994 & 1.67437 & -0.38346 \\
\hline & 5 & 1.50449 & -0.29485 & 1.53614 & -0.32743 & 1.45456 & -0.29843 \\
\hline \multirow{6}{*}{8} & 1 & 1.96537 & -0.56323 & 1.95270 & -0.56142 & 1.95288 & -0.56163 \\
\hline & 2 & 1.92479 & -0.52065 & 1.91298 & -0.52285 & 1.90898 & -0.51950 \\
\hline & 3 & 1.86766 & -0.47549 & 1.83779 & -0.46865 & 1.84663 & -0.47270 \\
\hline & 4 & 1.77636 & -0.41916 & 1.77284 & -0.43123 & 1.75654 & -0.42269 \\
\hline & 5 & 1.64785 & -0.35591 & 1.65752 & -0.37605 & 1.62040 & -0.36036 \\
\hline & 6 & 1.43326 & -0.27115 & 1.47800 & -0.30651 & 1.39558 & -0.27889 \\
\hline \multirow{7}{*}{9} & 1 & 1.96979 & -0.57064 & 1.95863 & -0.56838 & 1.95988 & -0.56989 \\
\hline & 2 & 1.93576 & -0.53244 & 1.92511 & -0.53351 & 1.92395 & -0.53246 \\
\hline & 3 & 1.88952 & -0.49297 & 1.88015 & -0.49717 & 1.87488 & -0.49338 \\
\hline & 4 & 1.82588 & -0.44958 & 1.81952 & -0.45748 & 1.80735 & -0.45034 \\
\hline & 5 & 1.73608 & -0.39956 & 1.73608 & -0.41237 & 1.71209 & -0.40077 \\
\hline & 6 & 1.60303 & -0.33858 & 1.61677 & -0.35886 & 1.57737 & -0.34312 \\
\hline & 7 & 1.38549 & -0.25722 & 1.32181 & -0.29151 & 1.34407 & -0.26268 \\
\hline \multirow{8}{*}{10} & 1 & 1.97368 & -0.57735 & 1.96373 & -0.57476 & 1.96506 & -0.57649 \\
\hline & 2 & 1.94494 & -0.54287 & 1.93525 & -0.54308 & 1.93487 & -0.5427 \\
\hline & 3 & 1.90697 & -0.50797 & 1.89806 & -0.51069 & 1.89489 & -0.50822 \\
\hline & 4 & 1.85793 & -0.47124 & 1.84941 & -0.47607 & 1.84165 & -0.47108 \\
\hline & 5 & 1.78869 & -0.42895 & 1.78506 & -0.43782 & 1.76991 & -0.42967 \\
\hline & 6 & 1.69455 & -0.38063 & 1.69791 & -0.39414 & 1.67028 & -0.38164 \\
\hline & 7 & 1.55743 & -0.32171 & 1.57513 & -0.34224 & 1.52547 & -0.32349 \\
\hline & 8 & 1.3371 & -0.24336 & 1.38993 & -0.27708 & 1.29554 & -0.24805 \\
\hline
\end{tabular}




\section{ESTIMATION FROM SAMPLES IN EXPONENTIAL \& GAMMA MODELS}

Table 4. Gamma (2) distribution empirical sample characteristics of MMLEs of $\sigma$ from right censored sample

\begin{tabular}{l|l|cccc}
\hline & & \multicolumn{3}{c}{ VAR } & MSE \\
\cline { 2 - 6 } $\boldsymbol{n}$ & $\boldsymbol{r}$ & EXACT MLE & EXT MLE & BLUE & EXACT MLE \\
\hline $\mathbf{3}$ & $\mathbf{1}$ & 0.00975 & 0.22591 & 0.22803 & 0.22600 \\
\hline $\mathbf{4}$ & $\mathbf{1}$ & 0.00277 & 0.15943 & 0.15510 & 0.15944 \\
& $\mathbf{2}$ & 0.01334 & 0.21390 & 0.21523 & 0.21408 \\
\hline & $\mathbf{1}$ & 0.00563 & 0.12188 & 0.11782 & 0.12191 \\
$\mathbf{5}$ & $\mathbf{2}$ & 0.00281 & 0.15087 & 0.14766 & 0.15088 \\
& $\mathbf{3}$ & 0.02190 & 0.19516 & 0.20653 & 0.19564 \\
\hline & $\mathbf{1}$ & 0.00870 & 0.09425 & 0.09509 & 0.09433 \\
$\mathbf{6}$ & $\mathbf{2}$ & 0.00771 & 0.11231 & 0.11285 & 0.11237 \\
& $\mathbf{3}$ & 0.01401 & 0.14175 & 0.14221 & 0.14194 \\
& $\mathbf{4}$ & 0.02220 & 0.18893 & 0.20008 & 0.18942 \\
\hline & $\mathbf{1}$ & 0.00963 & 0.07646 & 0.07975 & 0.07655 \\
& $\mathbf{2}$ & 0.00852 & 0.08823 & 0.09154 & 0.08830 \\
$\mathbf{7}$ & $\mathbf{3}$ & 0.01057 & 0.10779 & 0.10913 & 0.10790 \\
& $\mathbf{4}$ & 0.01027 & 0.13416 & 0.13807 & 0.13426 \\
& $\mathbf{5}$ & 0.02869 & 0.17353 & 0.19505 & 0.17435 \\
\hline & $\mathbf{1}$ & 0.00434 & 0.06593 & 0.06870 & 0.06594 \\
& $\mathbf{2}$ & 0.01160 & 0.07706 & 0.07708 & 0.07720 \\
$\mathbf{8}$ & $\mathbf{3}$ & 0.00288 & 0.08631 & 0.08878 & 0.08632 \\
& $\mathbf{4}$ & 0.00109 & 0.10277 & 0.10614 & 0.10277 \\
& $\mathbf{5}$ & 0.01748 & 0.12868 & 0.13469 & 0.12899 \\
& $\mathbf{6}$ & 0.01791 & 0.19094 & 0.19103 & 0.19126 \\
\hline & $\mathbf{1}$ & 0.00464 & 0.06160 & 0.06035 & 0.06163 \\
& $\mathbf{2}$ & 0.00044 & 0.06707 & 0.06661 & 0.06707 \\
& $\mathbf{3}$ & 0.00442 & 0.07414 & 0.07495 & 0.07416 \\
$\mathbf{9}$ & $\mathbf{4}$ & 0.00048 & 0.08776 & 0.08654 & 0.08776 \\
& $\mathbf{5}$ & 0.00136 & 0.10196 & 0.10372 & 0.10196 \\
& $\mathbf{6}$ & 0.01426 & 0.12706 & 0.13196 & 0.12726 \\
& $\mathbf{7}$ & 0.02398 & 0.17928 & 0.18767 & 0.17986 \\
\hline & $\mathbf{1}$ & 0.00018 & 0.05285 & 0.05382 & 0.05285 \\
& $\mathbf{2}$ & 0.00243 & 0.05726 & 0.05867 & 0.05726 \\
& $\mathbf{3}$ & 0.00086 & 0.06598 & 0.06492 & 0.06598 \\
& $\mathbf{4}$ & 0.00204 & 0.07066 & 0.07319 & 0.07067 \\
& $\mathbf{5}$ & 0.00035 & 0.08212 & 0.08465 & 0.08212 \\
& $\mathbf{6}$ & 0.01394 & 0.09408 & 0.10168 & 0.09428 \\
& $\mathbf{7}$ & 0.00585 & 0.12892 & 0.12963 & 0.12895 \\
& & 0.03092 & 0.17385 & 0.18487 & 0.17481 \\
\hline
\end{tabular}




\section{KANTAM \& SRIRAM}

Table 5. Gamma (2) distribution empirical sample characteristics of MMLEs of $\sigma$ from left censored sample

\begin{tabular}{|c|c|c|c|c|c|c|c|c|c|c|c|c|c|c|}
\hline \multirow[b]{2}{*}{$n$} & \multirow[b]{2}{*}{$r$} & \multicolumn{4}{|c|}{ BIAS } & \multicolumn{5}{|c|}{ VAR } & \multicolumn{4}{|c|}{ MSE } \\
\hline & & $\sigma_{M}$ & $\sigma_{1}$ & $\sigma_{2}$ & $\sigma_{3}$ & $\sigma_{M}$ & $\sigma_{B}$ & $\sigma_{1}$ & $\sigma_{2}$ & $\sigma_{3}$ & $\sigma_{M}$ & $\sigma_{1}$ & $\sigma_{2}$ & $\sigma_{3}$ \\
\hline 3 & 1 & 0.00029 & 0.00312 & 0.00893 & 0.00874 & 0.17905 & 0.17425 & 0.17425 & 0.17427 & 0.17426 & 0.17905 & 0.17629 & 0.17748 & 0.17739 \\
\hline \multirow{2}{*}{4} & 1 & 0.01058 & 0.00088 & 0.00381 & 0.00378 & 0.13410 & 0.12758 & 0.12758 & 0.12757 & 0.12758 & 0.13422 & 0.12735 & 0.12856 & 0.12855 \\
\hline & 2 & 0.00624 & 0.00435 & 0.01450 & 0.01408 & 0.13459 & 0.13744 & 0.13745 & 0.13749 & 0.13746 & 0.13463 & 0.13628 & 0.14172 & 0.14156 \\
\hline \multirow{3}{*}{5} & 1 & 0.00050 & 0.00040 & 0.00204 & 0.00203 & 0.10704 & 0.10115 & 0.10118 & 0.10323 & 0.12016 & 0.10704 & 0.10110 & 0.10366 & 0.12065 \\
\hline & 2 & 0.00016 & 0.00120 & 0.00670 & 0.00668 & 0.10403 & 0.10489 & 0.10491 & 0.11263 & 0.11227 & 0.10403 & 0.10466 & 0.11420 & 0.11382 \\
\hline & 3 & 0.02087 & 0.00510 & 0.01820 & 0.01759 & 0.12203 & 0.11552 & 0.11547 & 0.12794 & 0.12668 & 0.12247 & 0.11433 & 0.13296 & 0.13148 \\
\hline \multirow{4}{*}{6} & 1 & 0.01104 & 0.00023 & 0.00125 & 0.00125 & 0.08554 & 0.08394 & 0.08394 & 0.08394 & 0.08394 & 0.08566 & 0.08390 & 0.08415 & 0.08414 \\
\hline & 2 & 0.00055 & 0.00053 & 0.00381 & 0.00378 & 0.08389 & 0.08575 & 0.08575 & 0.08575 & 0.08575 & 0.08389 & 0.08566 & 0.08643 & 0.08641 \\
\hline & 3 & 0.00691 & 0.00409 & 0.00891 & 0.00876 & 0.09437 & 0.09008 & 0.08963 & 0.09009 & 0.09008 & 0.09442 & 0.08891 & 0.09178 & 0.09175 \\
\hline & 4 & 0.01417 & 0.00568 & 0.02067 & 0.01998 & 0.10788 & 0.10087 & 0.10088 & 0.10093 & 0.10089 & 0.10808 & 0.09976 & 0.10557 & 0.10536 \\
\hline \multirow{5}{*}{7} & 1 & 0.00943 & 0.00015 & 0.00083 & 0.00083 & 0.07002 & 0.07178 & 0.07179 & 0.07178 & 0.07178 & 0.07011 & 0.07178 & 0.07190 & 0.07190 \\
\hline & 2 & 0.00152 & 0.00031 & 0.00241 & 0.00241 & 0.07475 & 0.07279 & 0.07278 & 0.07278 & 0.07278 & 0.07475 & 0.07274 & 0.07314 & 0.07314 \\
\hline & 3 & 0.00479 & 0.00063 & 0.00523 & 0.00518 & 0.07750 & 0.07497 & 0.07497 & 0.07498 & 0.07497 & 0.07753 & 0.07488 & 0.07579 & 0.07578 \\
\hline & 4 & 0.00659 & 0.00163 & 0.01057 & 0.01036 & 0.08170 & 0.07958 & 0.07958 & 0.07959 & 0.07958 & 0.08174 & 0.07932 & 0.08140 & 0.08134 \\
\hline & 5 & 0.01722 & 0.00495 & 0.02249 & 0.02174 & 0.09591 & 0.09093 & 0.09034 & 0.09039 & 0.09052 & 0.09620 & 0.08947 & 0.09501 & 0.09479 \\
\hline
\end{tabular}




\section{ESTIMATION FROM SAMPLES IN EXPONENTIAL \& GAMMA MODELS}

Table 5 (contd.). Gamma (2) distribution empirical sample characteristics of MMLEs of $\sigma$ from left censored sample

\begin{tabular}{|c|c|c|c|c|c|c|c|c|c|c|c|c|c|c|}
\hline \multirow[b]{2}{*}{$n$} & \multicolumn{5}{|c|}{ BIAS } & \multicolumn{5}{|c|}{ VAR } & \multicolumn{4}{|c|}{ MSE } \\
\hline & $r$ & $\sigma_{M}$ & $\sigma_{1}$ & $\sigma_{2}$ & $\sigma_{3}$ & $\sigma_{M}$ & $\sigma_{B}$ & $\sigma_{1}$ & $\sigma_{2}$ & $\sigma_{3}$ & $\sigma_{M}$ & $\sigma_{1}$ & $\sigma_{2}$ & $\sigma_{3}$ \\
\hline \multirow{6}{*}{8} & 1 & 0.00487 & 0.00010 & 0.00059 & 0.00059 & 0.06025 & 0.06273 & 0.06127 & 0.06273 & 0.06273 & 0.06027 & 0.06126 & 0.06280 & 0.06280 \\
\hline & 2 & 0.00484 & 0.00017 & 0.00165 & 0.00165 & 0.06430 & 0.06393 & 0.06333 & 0.06334 & 0.06333 & 0.06432 & 0.06331 & 0.06355 & 0.06355 \\
\hline & 3 & 0.00107 & 0.00030 & 0.00342 & 0.00339 & 0.06424 & 0.06459 & 0.06459 & 0.06461 & 0.06459 & 0.06424 & 0.06455 & 0.06504 & 0.06504 \\
\hline & 4 & 0.00773 & 0.00079 & 0.00636 & 0.00196 & 0.06513 & 0.06699 & 0.06699 & 0.06700 & 0.06758 & 0.06519 & 0.06683 & 0.06789 & 0.06785 \\
\hline & 5 & 0.00540 & 0.00193 & 0.01179 & 0.01161 & 0.07190 & 0.07173 & 0.07173 & 0.07175 & 0.07173 & 0.07193 & 0.07145 & 0.07359 & 0.07355 \\
\hline & 6 & 0.02547 & 0.00672 & 0.02374 & 0.02308 & 0.08639 & 0.08295 & 0.08235 & 0.08241 & 0.08236 & 0.08704 & 0.08129 & 0.08693 & 0.08674 \\
\hline \multirow{4}{*}{9} & 1 & 0.00389 & 0.00008 & 0.00044 & 0.00044 & 0.05602 & 0.05571 & 0.05571 & 0.05571 & 0.05571 & 0.05604 & 0.05569 & 0.05576 & 0.05575 \\
\hline & 2 & 0.00179 & 0.00013 & 0.00119 & 0.00119 & 0.05655 & 0.05610 & 0.05610 & 0.05610 & 0.05610 & 0.05655 & 0.05608 & 0.05624 & 0.05624 \\
\hline & 3 & 0.00049 & 0.00021 & 0.00238 & 0.00238 & 0.05533 & 0.05689 & 0.05689 & 0.05689 & 0.05689 & 0.05533 & 0.05686 & 0.05716 & 0.05716 \\
\hline & 4 & 0.00800 & 0.00039 & 0.00425 & 0.00422 & 0.05885 & 0.05891 & 0.05831 & 0.05831 & 0.05831 & 0.05891 & 0.05826 & 0.05883 & 0.05882 \\
\hline \multirow{5}{*}{10} & 5 & 0.00482 & 0.00077 & 0.00733 & 0.00723 & 0.06151 & 0.06084 & 0.06085 & 0.06085 & 0.06085 & 0.06154 & 0.06075 & 0.06180 & 0.06178 \\
\hline & 6 & 0.00731 & 0.00191 & 0.01288 & 0.01258 & 0.06874 & 0.06563 & 0.06563 & 0.06565 & 0.06563 & 0.06880 & 0.06538 & 0.06752 & 0.06746 \\
\hline & 7 & 0.02974 & 0.00662 & 0.08570 & 0.02413 & 0.08059 & 0.07607 & 0.07607 & 0.07613 & 0.07608 & 0.08147 & 0.07512 & 0.09708 & 0.08038 \\
\hline & 1 & 0.00178 & 0.00003 & 0.00036 & 0.00036 & 0.04805 & 0.05011 & 0.05010 & 0.05059 & 0.05059 & 0.04805 & 0.05010 & 0.05063 & 0.05063 \\
\hline & 2 & 0.00181 & 0.00005 & 0.00095 & 0.00095 & 0.04765 & 0.05038 & 0.05036 & 0.05258 & 0.05258 & 0.04766 & 0.05036 & 0.05268 & 0.05268 \\
\hline
\end{tabular}




\section{KANTAM \& SRIRAM}

Table 6. Exponential distribution constants of linear approximation for MMLEs from left censored sample

\begin{tabular}{|c|c|c|c|c|c|c|c|}
\hline \multirow[b]{2}{*}{$n$} & \multirow[b]{2}{*}{$r$} & \multicolumn{2}{|c|}{ Method I } & \multicolumn{2}{|c|}{ Method II } & \multicolumn{2}{|c|}{ Method III } \\
\hline & & $\alpha$ & $\beta$ & $\alpha$ & $\beta$ & $\alpha$ & $\beta$ \\
\hline 3 & 1 & 0.97063 & -0.35651 & 0.96090 & -0.38629 & 0.94408 & -0.36425 \\
\hline \multirow{2}{*}{4} & 1 & 0.98576 & -0.40146 & 0.97853 & -0.41559 & 0.97211 & -0.40386 \\
\hline & 2 & 0.93657 & -0.31895 & 0.93287 & -0.35143 & 0.90767 & -0.32622 \\
\hline \multirow{3}{*}{5} & 1 & 0.99154 & -0.42443 & 0.98640 & -0.43278 & 0.98329 & -0.42550 \\
\hline & 2 & 0.96653 & -0.36966 & 0.96091 & -0.38629 & 0.95039 & -0.37206 \\
\hline & 3 & 0.90325 & -0.29059 & 0.90521 & -0.32396 & 0.87336 & -0.29720 \\
\hline \multirow{4}{*}{6} & 1 & 0.99438 & -0.43859 & 0.99062 & -0.44413 & 0.98887 & -0.43916 \\
\hline & 2 & 0.97914 & -0.39740 & 0.97431 & -0.40769 & 0.96890 & -0.39851 \\
\hline & 3 & 0.94575 & -0.34405 & 0.94226 & -0.36208 & 0.92807 & -0.34628 \\
\hline & 4 & 0.87203 & -0.26813 & 0.87888 & -0.30155 & 0.84178 & -0.27408 \\
\hline \multirow{5}{*}{7} & 1 & 0.99600 & -0.44824 & 0.99313 & -0.45218 & 0.99166 & -0.44857 \\
\hline & 2 & 0.98570 & -0.41520 & 0.98179 & -0.42224 & 0.97864 & -0.41581 \\
\hline & 3 & 0.96487 & -0.37474 & 0.96090 & -0.38629 & 0.95328 & -0.37579 \\
\hline & 4 & 0.92481 & -0.32278 & 0.92354 & -0.34159 & 0.90617 & -0.32484 \\
\hline & 5 & 0.85945 & -0.26815 & 0.85414 & -0.28279 & 0.81291 & -0.25510 \\
\hline \multirow{6}{*}{8} & 1 & 0.99711 & -0.45577 & 0.99487 & -0.45867 & 0.99404 & -0.45546 \\
\hline & 2 & 0.98988 & -0.42856 & 0.98674 & -0.43361 & 0.98441 & -0.42803 \\
\hline & 3 & 0.97598 & -0.39663 & 0.97245 & -0.40443 & 0.96711 & -0.39564 \\
\hline & 4 & 0.95142 & -0.35785 & 0.94852 & -0.36968 & 0.93727 & -0.35632 \\
\hline & 5 & 0.90254 & -0.30299 & 0.90357 & -0.32247 & 0.88505 & -0.30655 \\
\hline & 6 & 0.81412 & -0.23256 & 0.82901 & -0.26546 & 0.78652 & -0.23915 \\
\hline \multirow{6}{*}{9} & 1 & 0.99767 & -0.46057 & 0.99586 & -0.46287 & 0.99537 & -0.46072 \\
\hline & 2 & 0.99206 & -0.43689 & 0.98947 & -0.44081 & 0.98812 & -0.43714 \\
\hline & 3 & 0.98165 & -0.40959 & 0.97853 & -0.41559 & 0.97555 & -0.40995 \\
\hline & 4 & 0.96397 & -0.37744 & 0.96091 & -0.38629 & 0.95493 & -0.37798 \\
\hline & 5 & 0.93461 & -0.33850 & 0.93287 & -0.35143 & 0.92133 & -0.33935 \\
\hline & 6 & 0.88472 & -0.28916 & 0.88748 & -0.30855 & 0.86495 & -0.29076 \\
\hline \multirow{9}{*}{10} & 7 & 0.79223 & -0.22112 & 0.80946 & -0.25295 & 0.76233 & -0.22551 \\
\hline & 1 & 0.99815 & -0.46478 & 0.99666 & -0.46662 & 0.99630 & -0.46488 \\
\hline & 2 & 0.99374 & -0.44402 & 0.99158 & -0.44710 & 0.99063 & -0.44420 \\
\hline & 3 & 0.98582 & -0.42056 & 0.98315 & -0.42518 & 0.98111 & -0.42079 \\
\hline & 4 & 0.97280 & -0.39354 & 0.96993 & -0.40019 & 0.96597 & -0.39387 \\
\hline & 5 & 0.95220 & -0.36188 & 0.94976 & -0.37125 & 0.94247 & -0.36234 \\
\hline & 6 & 0.91952 & -0.32366 & 0.91892 & -0.33695 & 0.90570 & -0.32440 \\
\hline & 7 & 0.86594 & -0.27553 & 0.87044 & -0.29494 & 0.84590 & -0.27695 \\
\hline & 8 & 0.76974 & -0.20972 & 0.78932 & -0.24079 & 0.74009 & -0.21367 \\
\hline
\end{tabular}




\section{ESTIMATION FROM SAMPLES IN EXPONENTIAL \& GAMMA MODELS}

Table 7. Exponential distribution empirical sample characteristics of MMLEs of $\sigma$ from left censored sample

\begin{tabular}{|c|c|c|c|c|c|c|c|c|c|c|c|c|c|c|}
\hline \multirow[b]{2}{*}{$n$} & \multirow[b]{2}{*}{$r$} & \multicolumn{4}{|c|}{ BIAS } & \multicolumn{5}{|c|}{ VAR } & \multicolumn{4}{|c|}{ MSE } \\
\hline & & $\sigma_{\mathrm{M}}$ & $\sigma_{1}$ & $\sigma_{2}$ & $\sigma_{3}$ & $\sigma_{\mathrm{M}}$ & $\sigma_{\mathrm{B}}$ & $\sigma_{1}$ & $\sigma_{2}$ & $\sigma_{3}$ & $\sigma_{\mathrm{M}}$ & $\sigma_{1}$ & $\sigma_{2}$ & $\sigma_{3}$ \\
\hline 3 & 1 & 0.00703 & 0.00231 & 0.00934 & 0.00887 & 0.32718 & 0.34211 & 0.34214 & 0.34219 & 0.34215 & 0.32723 & 0.34056 & 0.34870 & 0.34833 \\
\hline \multirow{2}{*}{4} & 1 & 0.00041 & 0.00039 & 0.00349 & 0.00339 & 0.25367 & 0.25252 & 0.25253 & 0.25254 & 0.25253 & 0.25367 & 0.25233 & 0.25432 & 0.25426 \\
\hline & 2 & 0.02048 & 0.00398 & 0.01613 & 0.01523 & 0.27658 & 0.26521 & 0.26526 & 0.26537 & 0.26528 & 0.27699 & 0.26317 & 0.27262 & 0.27366 \\
\hline \multirow{3}{*}{5} & 1 & 0.00198 & 0.00011 & 0.00167 & 0.00164 & 0.20104 & 0.20098 & 0.20098 & 0.20098 & 0.20098 & 0.20104 & 0.20093 & 0.20165 & 0.20164 \\
\hline & 2 & 0.00613 & 0.00080 & 0.00678 & 0.00655 & 0.20473 & 0.20523 & 0.20525 & 0.20527 & 0.20525 & 0.20477 & 0.20492 & 0.20810 & 0.20798 \\
\hline & 3 & 0.01019 & 0.00520 & 0.02083 & 0.01963 & 0.22971 & 0.21966 & 0.21976 & 0.21984 & 0.21973 & 0.22981 & 0.21753 & 0.22952 & 0.22882 \\
\hline \multirow{4}{*}{6} & 1 & 0.00057 & 0.00004 & 0.00122 & 0.00092 & 0.16817 & 0.16712 & 0.16712 & 0.16702 & 0.16712 & 0.16817 & 0.16711 & 0.16743 & 0.16743 \\
\hline & 2 & 0.00737 & 0.00025 & 0.00351 & 0.00343 & 0.16318 & 0.16894 & 0.16895 & 0.16896 & 0.16895 & 0.16324 & 0.16887 & 0.17016 & 0.17013 \\
\hline & 3 & 0.00210 & 0.00115 & 0.00946 & 0.00911 & 0.16966 & 0.17425 & 0.17427 & 0.17431 & 0.17427 & 0.16967 & 0.17387 & 0.17771 & 0.17755 \\
\hline & 4 & 0.02233 & 0.00601 & 0.02419 & 0.02283 & 0.04620 & 0.18944 & 0.18949 & 0.18963 & 0.18951 & 0.20512 & 0.18725 & 0.19950 & 0.19878 \\
\hline \multirow{5}{*}{7} & 1 & 0.00628 & 0.00024 & 0.00058 & 0.00063 & 0.14681 & 0.14309 & 0.14309 & 0.14309 & 0.14309 & 0.14685 & 0.14302 & 0.14326 & 0.14327 \\
\hline & 2 & 0.00422 & 0.00008 & 0.00207 & 0.00203 & 0.14469 & 0.14401 & 0.14401 & 0.14401 & 0.14401 & 0.14471 & 0.14398 & 0.14461 & 0.14459 \\
\hline & 3 & 0.00313 & 0.00037 & 0.00518 & 0.00504 & 0.13967 & 0.14645 & 0.14645 & 0.14646 & 0.14645 & 0.13968 & 0.14634 & 0.14801 & 0.14796 \\
\hline & 4 & 0.00177 & 0.00143 & 0.01161 & 0.01117 & 0.15265 & 0.15239 & 0.15239 & 0.15245 & 0.15241 & 0.15265 & 0.15196 & 0.15614 & 0.15596 \\
\hline & 5 & 0.03487 & 0.00384 & 0.02668 & 0.02522 & 0.18565 & 0.16786 & 0.16796 & 0.16804 & 0.16791 & 0.18687 & 0.16927 & 0.17784 & 0.17712 \\
\hline
\end{tabular}




\section{KANTAM \& SRIRAM}

Table 7 (contd.). Exponential distribution empirical sample characteristics of MMLEs of $\sigma$ from left censored sample

\begin{tabular}{|c|c|c|c|c|c|c|c|c|c|c|c|c|c|c|}
\hline \multirow[b]{2}{*}{$n$} & \multirow[b]{2}{*}{$r$} & \multicolumn{4}{|c|}{ BIAS } & \multicolumn{5}{|c|}{ VAR } & \multicolumn{4}{|c|}{ MSE } \\
\hline & & $\sigma_{\mathrm{M}}$ & $\sigma_{1}$ & $\sigma_{2}$ & $\sigma_{3}$ & $\sigma_{\mathrm{M}}$ & $\sigma_{\mathrm{B}}$ & $\sigma_{1}$ & $\sigma_{2}$ & $\sigma_{3}$ & $\sigma_{\mathrm{M}}$ & $\sigma_{1}$ & $\sigma_{2}$ & $\sigma_{3}$ \\
\hline \multirow{6}{*}{8} & 1 & 0.00925 & 0.00000 & 0.00037 & 0.00037 & 0.12555 & 0.12514 & 0.12514 & 0.12514 & 0.12514 & 0.12563 & 0.12514 & 0.12523 & 0.12523 \\
\hline & 2 & 0.01269 & 0.00002 & 0.00132 & 0.00129 & 0.12342 & 0.12564 & 0.12565 & 0.12565 & 0.12565 & 0.12358 & 0.12564 & 0.12626 & 0.12597 \\
\hline & 3 & 0.00446 & 0.00004 & 0.00317 & 0.00309 & 0.12944 & 0.12693 & 0.12693 & 0.12694 & 0.12693 & 0.12946 & 0.12692 & 0.12775 & 0.12773 \\
\hline & 4 & 0.01985 & 0.00019 & 0.00667 & 0.00642 & 0.13718 & 0.12979 & 0.12981 & 0.12982 & 0.12981 & 0.13757 & 0.12976 & 0.13161 & 0.13152 \\
\hline & 5 & 0.01327 & 0.00188 & 0.01321 & 0.01279 & 0.14771 & 0.13612 & 0.13614 & 0.13619 & 0.13615 & 0.14789 & 0.13563 & 0.13998 & 0.13981 \\
\hline & 6 & 0.02799 & 0.00755 & 0.02836 & 0.02702 & 0.15657 & 0.15162 & 0.15166 & 0.15182 & 0.15168 & 0.15736 & 0.14943 & 0.16134 & 0.16071 \\
\hline \multirow{7}{*}{9} & 1 & 0.01059 & 0.00000 & 0.00026 & 0.00026 & 0.10773 & 0.11119 & 0.11119 & 0.11119 & 0.11119 & 0.10784 & 0.11119 & 0.11125 & 0.11125 \\
\hline & 2 & 0.00398 & 0.00002 & 0.00088 & 0.00087 & 0.10740 & 0.11150 & 0.11149 & 0.11149 & 0.11149 & 0.10741 & 0.11149 & 0.11169 & 0.11169 \\
\hline & 3 & 0.00990 & 0.00008 & 0.00207 & 0.00204 & 0.11348 & 0.11224 & 0.12224 & 0.11224 & 0.11224 & 0.11357 & 0.11222 & 0.11271 & 0.11270 \\
\hline & 4 & 0.00332 & 0.00023 & 0.00414 & 0.00406 & 0.10735 & 0.11381 & 0.11382 & 0.11382 & 0.11381 & 0.10736 & 0.11376 & 0.11478 & 0.11476 \\
\hline & 5 & 0.01077 & 0.00063 & 0.00781 & 0.00758 & 0.12049 & 0.11698 & 0.11698 & 0.11700 & 0.11698 & 0.12061 & 0.11684 & 0.11889 & 0.11883 \\
\hline & 6 & 0.01098 & 0.00189 & 0.01469 & 0.01412 & 0.12623 & 0.12353 & 0.12355 & 0.12359 & 0.12568 & 0.12635 & 0.12309 & 0.12747 & 0.12945 \\
\hline & 7 & 0.01943 & 0.00752 & 0.03000 & 0.02846 & 0.14248 & 0.13894 & 0.13897 & 0.13911 & 0.13898 & 0.14286 & 0.13695 & 0.14849 & 0.14785 \\
\hline \multirow{8}{*}{10} & 1 & 0.00435 & 0.00000 & 0.00018 & 0.00018 & 0.10156 & 0.10006 & 0.10006 & 0.10006 & 0.10006 & 0.10158 & 0.10003 & 0.10007 & 0.10007 \\
\hline & 2 & 0.00630 & 0.00001 & 0.00063 & 0.00062 & 0.09815 & 0.10025 & 0.10025 & 0.10025 & 0.10025 & 0.09819 & 0.10023 & 0.10035 & 0.10035 \\
\hline & 3 & 0.00134 & 0.00004 & 0.00143 & 0.00142 & 0.09606 & 0.10070 & 0.10071 & 0.10072 & 0.10071 & 0.09607 & 0.10071 & 0.10100 & 0.10100 \\
\hline & 4 & 0.01044 & 0.00011 & 0.00279 & 0.00275 & 0.10234 & 0.10164 & 0.10165 & 0.10166 & 0.10161 & 0.10245 & 0.10163 & 0.10224 & 0.10223 \\
\hline & 5 & 0.00136 & 0.00028 & 0.00503 & 0.00493 & 0.10330 & 0.10343 & 0.10344 & 0.10448 & 0.10344 & 0.10398 & 0.10338 & 0.10556 & 0.10448 \\
\hline & 6 & 0.01078 & 0.00072 & 0.00884 & 0.00857 & 0.11406 & 0.10680 & 0.10680 & 0.10681 & 0.10680 & 0.11418 & 0.10664 & 0.10879 & 0.10870 \\
\hline & 7 & 0.00377 & 0.00205 & 0.01584 & 0.01523 & 0.11753 & 0.11348 & 0.11349 & 0.11353 & 0.11348 & 0.11755 & 0.11302 & 0.11741 & 0.11720 \\
\hline & 8 & 0.02827 & 0.00780 & 0.03117 & 0.02961 & 0.13595 & 0.12873 & 0.12875 & 0.12888 & 0.12876 & 0.13674 & 0.12681 & 0.13801 & 0.13737 \\
\hline
\end{tabular}




\section{ESTIMATION FROM SAMPLES IN EXPONENTIAL \& GAMMA MODELS}

Table 8. Gamma (2) distribution empirical sample characteristic of reliability estimates in right censored samples

\begin{tabular}{|c|c|c|c|c|c|c|c|c|}
\hline \multirow[b]{2}{*}{$n$} & \multirow[b]{2}{*}{$r$} & \multirow[b]{2}{*}{$R(x)$} & \multicolumn{2}{|c|}{ BIAS } & \multicolumn{2}{|c|}{ VAR } & \multicolumn{2}{|c|}{ MSE } \\
\hline & & & MLE & BLUE & MLE & BLUE & MLE & BLUE \\
\hline 3 & 1 & 0.9 & 0.05736 & 0.05572 & 0.01769 & 0.01745 & 0.02098 & 0.02056 \\
\hline \multirow{2}{*}{4} & 1 & 0.9 & 0.03791 & 0.03717 & 0.00964 & 0.00957 & 0.01108 & 0.01095 \\
\hline & 2 & 0.9 & 0.05728 & 0.05449 & 0.01736 & 0.01697 & 0.02064 & 0.01994 \\
\hline \multirow{3}{*}{5} & 1 & 0.9 & 0.02948 & 0.02907 & 0.00660 & 0.00657 & 0.00747 & 0.00742 \\
\hline & 2 & 0.9 & 0.03647 & 0.03517 & 0.00925 & 0.00912 & 0.01058 & 0.01035 \\
\hline & 3 & 0.9 & 0.05637 & 0.05276 & 0.01764 & 0.01715 & 0.02082 & 0.01993 \\
\hline \multirow{4}{*}{6} & 1 & 0.9 & 0.01921 & 0.01896 & 0.00434 & 0.00433 & 0.00471 & 0.00469 \\
\hline & 2 & 0.9 & 0.02764 & 0.02690 & 0.00611 & 0.00605 & 0.00687 & 0.00678 \\
\hline & 3 & 0.9 & 0.03724 & 0.03549 & 0.00921 & 0.00904 & 0.01060 & 0.01030 \\
\hline & 4 & 0.9 & 0.05420 & 0.04995 & 0.01598 & 0.01541 & 0.01892 & 0.01790 \\
\hline \multirow{5}{*}{7} & 1 & 0.9 & 0.01917 & 0.01900 & 0.00353 & 0.00352 & 0.00389 & 0.00388 \\
\hline & 2 & 0.9 & 0.02165 & 0.02117 & 0.00406 & 0.00404 & 0.00453 & 0.00449 \\
\hline & 3 & 0.9 & 0.02779 & 0.02677 & 0.00602 & 0.00595 & 0.00679 & 0.00666 \\
\hline & 4 & 0.9 & 0.03534 & 0.03324 & 0.00907 & 0.00887 & 0.01031 & 0.00997 \\
\hline & 5 & 0.9 & 0.05240 & 0.04762 & 0.01491 & 0.01430 & 0.01765 & 0.01657 \\
\hline \multirow{6}{*}{8} & 1 & 0.9 & 0.01534 & 0.01522 & 0.00274 & 0.00274 & 0.00298 & 0.00297 \\
\hline & 2 & 0.9 & 0.01915 & 0.01882 & 0.00328 & 0.00326 & 0.00365 & 0.00362 \\
\hline & 3 & 0.9 & 0.02042 & 0.01976 & 0.00435 & 0.00431 & 0.00477 & 0.00470 \\
\hline & 4 & 0.9 & 0.02414 & 0.02290 & 0.00538 & 0.00530 & 0.00597 & 0.00582 \\
\hline & 5 & 0.9 & 0.03573 & 0.03330 & 0.00862 & 0.00841 & 0.00990 & 0.00951 \\
\hline & 6 & 0.9 & 0.05261 & 0.04739 & 0.01540 & 0.01471 & 0.01816 & 0.01695 \\
\hline \multirow{7}{*}{9} & 1 & 0.9 & 0.01248 & 0.01238 & 0.00238 & 0.00238 & 0.00253 & 0.00253 \\
\hline & 2 & 0.9 & 0.01499 & 0.01475 & 0.00284 & 0.00283 & 0.00307 & 0.00305 \\
\hline & 3 & 0.9 & 0.01760 & 0.01714 & 0.00331 & 0.00329 & 0.00362 & 0.00358 \\
\hline & 4 & 0.9 & 0.01982 & 0.01900 & 0.00416 & 0.00411 & 0.00455 & 0.00447 \\
\hline & 5 & 0.9 & 0.02368 & 0.02224 & 0.00506 & 0.00497 & 0.00562 & 0.00546 \\
\hline & 6 & 0.9 & 0.03404 & 0.03136 & 0.00787 & 0.00764 & 0.00903 & 0.00862 \\
\hline & 7 & 0.9 & 0.05323 & 0.04765 & 0.01601 & 0.01528 & 0.01884 & 0.01755 \\
\hline \multirow{8}{*}{10} & 1 & 0.9 & 0.01131 & 0.01124 & 0.00193 & 0.00192 & 0.00205 & 0.00205 \\
\hline & 2 & 0.9 & 0.01285 & 0.01267 & 0.00219 & 0.00219 & 0.00236 & 0.00235 \\
\hline & 3 & 0.9 & 0.01493 & 0.01459 & 0.00281 & 0.00280 & 0.00303 & 0.00301 \\
\hline & 4 & 0.9 & 0.01612 & 0.01553 & 0.00301 & 0.00299 & 0.00327 & 0.00323 \\
\hline & 5 & 0.9 & 0.01874 & 0.01778 & 0.00380 & 0.00375 & 0.00415 & 0.00407 \\
\hline & 6 & 0.9 & 0.02543 & 0.02379 & 0.00534 & 0.00523 & 0.00598 & 0.00579 \\
\hline & 7 & 0.9 & 0.03207 & 0.02919 & 0.00772 & 0.00747 & 0.00875 & 0.00832 \\
\hline & 8 & 0.9 & 0.05336 & 0.04741 & 0.01511 & 0.01435 & 0.01796 & 0.01660 \\
\hline
\end{tabular}




\section{KANTAM \& SRIRAM}

Table 9. Gamma (2) distribution empirical sample characteristic of reliability estimates in left censored samples

\begin{tabular}{|c|c|c|c|c|c|c|c|c|c|c|c|c|c|c|c|c|c|}
\hline \multirow[b]{2}{*}{$n$} & \multirow[b]{2}{*}{$r$} & \multirow[b]{2}{*}{$R(x)$} & \multicolumn{5}{|c|}{ BIAS } & \multicolumn{5}{|c|}{ VARIANCE } & \multicolumn{5}{|c|}{ MSE } \\
\hline & & & MLE & MET. I & MET. II & MET. III & BLUE & MLE & MET. I & MET. II & MET. III & BLUE & MLE & MET. I & MET. II & MET. III & BLUE \\
\hline 3 & 1 & 0.9 & 0.04216 & 0.04425 & 0.04173 & 0.04177 & 0.04360 & 0.01196 & 0.01220 & 0.01192 & 0.01192 & 0.01213 & 0.01374 & 0.01416 & 0.01366 & 0.01367 & 0.01403 \\
\hline \multirow{2}{*}{4} & 1 & 0.9 & 0.02725 & 0.02787 & 0.02696 & 0.02696 & 0.02769 & 0.00656 & 0.00661 & 0.00654 & 0.00654 & 0.00660 & 0.00731 & 0.00739 & 0.00727 & 0.00727 & 0.00737 \\
\hline & 2 & 0.9 & 0.02912 & 0.03225 & 0.02855 & 0.02861 & 0.03137 & 0.00715 & 0.00741 & 0.00711 & 0.00711 & 0.00733 & 0.00800 & 0.00845 & 0.00792 & 0.00793 & 0.00832 \\
\hline \multirow{3}{*}{5} & 1 & 0.9 & 0.02414 & 0.02443 & 0.02396 & 0.02396 & 0.02097 & 0.00513 & 0.00515 & 0.00512 & 0.00512 & 0.00494 & 0.00571 & 0.00575 & 0.00569 & 0.00569 & 0.00538 \\
\hline & 2 & 0.9 & 0.02355 & 0.02469 & 0.02315 & 0.02317 & 0.02078 & 0.00512 & 0.00520 & 0.00510 & 0.00510 & 0.00495 & 0.00567 & 0.00581 & 0.00563 & 0.00563 & 0.00538 \\
\hline & 3 & 0.9 & 0.02171 & 0.02554 & 0.02111 & 0.02121 & 0.02063 & 0.00521 & 0.00547 & 0.00516 & 0.00517 & 0.00513 & 0.00568 & 0.00612 & 0.00561 & 0.00562 & 0.00556 \\
\hline \multirow{4}{*}{6} & 1 & 0.9 & 0.01654 & 0.01669 & 0.01642 & 0.01642 & 0.01665 & 0.00364 & 0.00365 & 0.00364 & 0.00364 & 0.00365 & 0.00392 & 0.00393 & 0.00391 & 0.00391 & 0.00392 \\
\hline & 2 & 0.9 & 0.01845 & 0.01898 & 0.01817 & 0.01817 & 0.01888 & 0.00368 & 0.00370 & 0.00366 & 0.00366 & 0.00370 & 0.00402 & 0.00406 & 0.00399 & 0.00399 & 0.00405 \\
\hline & 3 & 0.9 & 0.01915 & 0.02167 & 0.01872 & 0.01875 & 0.02039 & 0.00406 & 0.00420 & 0.00404 & 0.00405 & 0.00414 & 0.00443 & 0.00467 & 0.00439 & 0.00440 & 0.00455 \\
\hline & 4 & 0.9 & 0.02053 & 0.02494 & 0.01992 & 0.02006 & 0.02385 & 0.00467 & 0.00495 & 0.00463 & 0.00465 & 0.00489 & 0.00509 & 0.00557 & 0.00503 & 0.00505 & 0.00546 \\
\hline \multirow{5}{*}{7} & 1 & 0.9 & 0.01762 & 0.01771 & 0.01753 & 0.01753 & 0.01769 & 0.00316 & 0.00316 & 0.00315 & 0.00315 & 0.00316 & 0.00347 & 0.00347 & 0.00346 & 0.00346 & 0.00347 \\
\hline & 2 & 0.9 & 0.01591 & 0.01622 & 0.01571 & 0.01571 & 0.01615 & 0.00296 & 0.00297 & 0.00295 & 0.00295 & 0.00297 & 0.00321 & 0.00324 & 0.00320 & 0.00320 & 0.00323 \\
\hline & 3 & 0.9 & 0.01612 & 0.01686 & 0.01578 & 0.01579 & 0.01674 & 0.00324 & 0.00327 & 0.00322 & 0.00322 & 0.00326 & 0.00350 & 0.00355 & 0.00347 & 0.00347 & 0.00355 \\
\hline & 4 & 0.9 & 0.01644 & 0.01824 & 0.01598 & 0.01602 & 0.01794 & 0.00336 & 0.00345 & 0.00334 & 0.00334 & 0.00343 & 0.00363 & 0.00378 & 0.00359 & 0.00360 & 0.00376 \\
\hline & 5 & 0.9 & 0.01657 & 0.02104 & 0.01596 & 0.01609 & 0.02009 & 0.00364 & 0.00387 & 0.00361 & 0.00362 & 0.00383 & 0.00392 & 0.00432 & 0.00387 & 0.00388 & 0.00423 \\
\hline
\end{tabular}




\section{ESTIMATION FROM SAMPLES IN EXPONENTIAL \& GAMMA MODELS}

Table 9 (contd.). Gamma (2) distribution empirical sample characteristic of reliability estimates in left censored samples

\begin{tabular}{|c|c|c|c|c|c|c|c|c|c|c|c|c|c|c|c|c|c|}
\hline \multirow[b]{2}{*}{$n$} & \multirow[b]{2}{*}{$r$} & \multirow[b]{2}{*}{$R(x)$} & \multicolumn{5}{|c|}{ BIAS } & \multicolumn{5}{|c|}{ VARIANCE } & \multicolumn{5}{|c|}{ MSE } \\
\hline & & & MLE & MET. I & MET. II & MET. III & BLUE & MLE & MET. I & MET. II & MET. III & BLUE & MLE & MET. I & MET. II & MET. III & BLUE \\
\hline \multirow{6}{*}{8} & 1 & 0.9 & .01405 & 1411 & & 01398 & 0.01409 & 0239 & 00240 & 0239 & 0239 & 00240 & 0259 & 00259 & & 0259 & \\
\hline & 2 & 0.9 & 0.01484 & 0.01503 & 0.01469 & 0.01469 & 0.01499 & 0.00254 & 0.00255 & 0.00254 & 0.00254 & 0.00255 & 0.00277 & 0.00278 & 0.00275 & 0.00275 & 0.00278 \\
\hline & 3 & 0.9 & 0.01412 & 0.01455 & 0.01387 & 0.01387 & 0.01450 & 0.00273 & 0.00275 & 0.00272 & 0.00272 & 0.00275 & 0.00293 & 0.00296 & 0.00291 & 0.00291 & 0.00296 \\
\hline & 4 & 0.9 & 0.01299 & 0.01395 & 0.01266 & 0.01267 & 0.01382 & 0.00270 & 0.00274 & 0.00269 & 0.00269 & 0.00273 & 0.00287 & 0.00293 & 0.00285 & 0.00285 & 0.00292 \\
\hline & 5 & 0.9 & 0.01440 & 0.01647 & 0.01397 & 0.01399 & 0.01611 & 0.00279 & 0.00287 & 0.00277 & 0.00277 & 0.00286 & 0.00300 & 0.00314 & 0.00297 & 0.00297 & 0.00312 \\
\hline & 6 & 0.9 & 0.01294 & 0.01795 & 0.01241 & 0.01253 & 0.01670 & 0.00313 & 0.00336 & 0.00311 & 0.00312 & 0.00331 & 0.00330 & 0.00369 & 0.00326 & 0.00327 & 0.00359 \\
\hline \multirow{7}{*}{9} & 1 & 0.9 & 01136 & 0.01140 & 0.01130 & 0.01130 & 0.01138 & 0.00210 & 0.00210 & 0.00210 & 0.00210 & 0.00210 & 0.00223 & 0.00223 & 0.00223 & 0.00223 & 0.00223 \\
\hline & 2 & 0.9 & 0.01205 & 0.01217 & 0.01194 & 0.01194 & 0.01215 & 0.00222 & 0.00222 & 0.00221 & 0.00221 & 0.00222 & 0.00236 & 0.00237 & 0.00235 & 0.00235 & 0.00237 \\
\hline & 3 & 0.9 & 0.01201 & 0.01228 & 0.01182 & 0.01182 & 0.01224 & 0.00214 & 0.00215 & 0.00213 & 0.00213 & 0.00215 & 0.00228 & 0.00230 & 0.00227 & 0.00227 & 0.00230 \\
\hline & 4 & 0.9 & 0.01122 & 0.01178 & 0.01095 & 0.01095 & 0.01170 & 0.00222 & 0.00224 & 0.00222 & 0.00222 & 0.00224 & 0.00235 & 0.00238 & 0.00234 & 0.00234 & 0.00238 \\
\hline & 5 & 0.9 & 0.01235 & 0.01346 & 0.01200 & 0.01202 & 0.01332 & 0.00235 & 0.00239 & 0.00234 & 0.00234 & 0.00239 & 0.00250 & 0.00257 & 0.00248 & 0.00248 & 0.00256 \\
\hline & 6 & 0.9 & 0.01326 & 0.01550 & 0.01281 & 0.01287 & 0.01515 & 0.00259 & 0.00268 & 0.00258 & 0.00258 & 0.00267 & 0.00277 & 0.00292 & 0.00274 & 0.00274 & 0.00290 \\
\hline & 7 & 0.9 & 0.01067 & 0.01575 & 0.00034 & 0.01025 & 0.01453 & 0.00265 & 0.00285 & 0.00225 & 0.00263 & 0.00280 & 0.00276 & 0.00310 & 0.00225 & 0.00274 & 0.00302 \\
\hline & 1 & 0.9 & 0.01058 & 0.01060 & 0.01053 & 0.01053 & 0.01059 & 0.00172 & 0.00172 & 0.00172 & 0.00172 & 0.00172 & 0.00183 & 0.00183 & 0.00183 & 0.00183 & 0.00183 \\
\hline & 2 & 0.9 & 0.01050 & 0.01058 & 0.01040 & 0.01040 & 0.01056 & 0.00172 & 0.00172 & 0.00172 & 0.00172 & 0.00172 & 0.00183 & 0.00183 & 0.00183 & 0.00183 & 0.00183 \\
\hline & 3 & 0.9 & 0.01159 & 0.01177 & 0.01143 & 0.01143 & 0.01174 & 0.00195 & 0.00196 & 0.00195 & 0.00195 & 0.00196 & 0.00209 & 0.00210 & 0.00208 & 0.00208 & 0.00209 \\
\hline & 4 & 0.9 & 0.01075 & 0.01114 & 0.01054 & 0.01055 & 0.01109 & 0.00188 & 0.00189 & 0.00187 & 0.00187 & 0.00189 & 0.00199 & 0.00201 & 0.00198 & 0.00198 & 0.00201 \\
\hline & 5 & 0.9 & 0.01075 & 0.01143 & 0.01047 & 0.01048 & 0.01136 & 0.00199 & 0.00201 & 0.00198 & 0.00198 & 0.00201 & 0.00211 & 0.00214 & 0.00209 & 0.00209 & 0.00214 \\
\hline & 6 & 0.9 & 0.01139 & 0.01262 & 0.01102 & 0.01104 & 0.01247 & 0.00208 & 0.00212 & 0.00207 & 0.00207 & 0.00211 & 0.00221 & 0.00228 & 0.00219 & 0.00219 & 0.00227 \\
\hline & 7 & 0.9 & 0.01114 & 0.01352 & 0.01069 & 0.01076 & 0.01316 & 0.00227 & 0.00235 & 0.00225 & 0.00226 & 0.00234 & 0.00239 & 0.00253 & 0.00237 & 0.00237 & 0.00251 \\
\hline & 8 & 0.9 & 0.01079 & 0.01613 & 0.01027 & 0.01042 & 0.01488 & 0.00263 & 0.00285 & 0.00261 & 0.00262 & 0.00280 & 0.00275 & 0.00311 & 0.00271 & 0.00273 & 0.00302 \\
\hline
\end{tabular}




\section{KANTAM \& SRIRAM}

Table 10. Exponential distribution empirical sample characteristic of reliability estimates in left censored samples

\begin{tabular}{|c|c|c|c|c|c|c|c|c|c|c|c|c|c|c|c|c|c|}
\hline \multirow[b]{2}{*}{$n$} & \multirow[b]{2}{*}{$r$} & \multirow[b]{2}{*}{$R(x)$} & \multicolumn{5}{|c|}{ BIAS } & \multicolumn{5}{|c|}{ VAR } & \multicolumn{5}{|c|}{ MSE } \\
\hline & & & MLE & MET. I & MET. II & MET. III & BLUE & MLE & MET. I & MET. II & MET. III & BLUE & MLE & MET. I & MET. II & MET. III & BLUE \\
\hline 3 & 1 & 0.9 & 0.03982 & 0.04103 & 0.03949 & 0.03955 & 0.03952 & 0.00954 & 0.00967 & 0.00951 & 0.00951 & 0.00935 & 0.01112 & 0.01135 & 0.01107 & 0.01108 & 0.01091 \\
\hline \multirow{2}{*}{4} & 1 & 0.9 & 0.02706 & 0.02734 & 0.02689 & 0.02690 & 0.02848 & 0.00517 & 0.00519 & 0.00516 & 0.00516 & 0.00575 & 0.00590 & 0.00594 & 0.00588 & 0.00588 & 0.00656 \\
\hline & 2 & 0.9 & 0.02632 & 0.02816 & 0.02586 & 0.02596 & 0.02821 & 0.00555 & 0.00568 & 0.00551 & 0.00552 & 0.00541 & 0.00624 & 0.00647 & 0.00618 & 0.00619 & 0.00621 \\
\hline \multirow{3}{*}{5} & 1 & 0.9 & 0.02079 & 0.02088 & 0.02068 & 0.02069 & 0.02335 & 0.00363 & 0.00363 & 0.00362 & 0.00362 & 0.00357 & 0.00406 & 0.00407 & 0.00405 & 0.00405 & 0.00411 \\
\hline & 2 & 0.9 & 0.02035 & 0.02091 & 0.02008 & 0.02010 & 0.02077 & 0.00347 & 0.00350 & 0.00346 & 0.00346 & 0.00383 & 0.00389 & 0.00394 & 0.00386 & 0.00387 & 0.00427 \\
\hline & 3 & 0.9 & 0.02212 & 0.02453 & 0.02162 & 0.02175 & 0.02082 & 0.00408 & 0.00422 & 0.00406 & 0.00406 & 0.00383 & 0.00457 & 0.00483 & 0.00452 & 0.00454 & 0.00427 \\
\hline \multirow{4}{*}{6} & 1 & 0.9 & 0.01727 & 0.01732 & 0.01721 & 0.01721 & 0.01784 & 0.00278 & 0.00278 & 0.00278 & 0.00278 & 0.00300 & 0.00308 & 0.00308 & 0.00308 & 0.00308 & 0.00332 \\
\hline & 2 & 0.9 & 0.01742 & 0.01765 & 0.01724 & 0.01725 & 0.01769 & 0.00261 & 0.00262 & 0.00261 & 0.00261 & 0.00271 & 0.00292 & 0.00293 & 0.00290 & 0.00290 & 0.00302 \\
\hline & 3 & 0.9 & 0.01739 & 0.01822 & 0.01707 & 0.01711 & 0.01875 & 0.00287 & 0.00290 & 0.00285 & 0.00286 & 0.00291 & 0.00317 & 0.00324 & 0.00314 & 0.00315 & 0.00326 \\
\hline & 4 & 0.9 & 0.01736 & 0.02010 & 0.01685 & 0.01698 & 0.02028 & 0.00310 & 0.00323 & 0.00307 & 0.00308 & 0.00320 & 0.00340 & 0.00363 & 0.00336 & 0.00337 & 0.00361 \\
\hline \multirow{5}{*}{7} & 1 & 0.9 & 0.01333 & 0.01335 & 0.01329 & 0.01329 & 0.01509 & 0.00196 & 0.00197 & 0.00196 & 0.00196 & 0.00226 & 0.00214 & 0.00214 & 0.00214 & 0.00214 & 0.00249 \\
\hline & 2 & 0.9 & 0.01493 & 0.01505 & 0.01482 & 0.01483 & 0.01542 & 0.00213 & 0.00214 & 0.00213 & 0.00213 & 0.00241 & 0.00236 & 0.00236 & 0.00235 & 0.00235 & 0.00265 \\
\hline & 3 & 0.9 & 0.01379 & 0.01415 & 0.01357 & 0.01358 & 0.01521 & 0.00206 & 0.00207 & 0.00205 & 0.00205 & 0.00202 & 0.00225 & 0.00227 & 0.00224 & 0.00224 & 0.00225 \\
\hline & 4 & 0.9 & 0.01510 & 0.01613 & 0.01474 & 0.01479 & 0.01417 & 0.00221 & 0.00225 & 0.00220 & 0.00220 & 0.00224 & 0.00244 & 0.00251 & 0.00242 & 0.00242 & 0.00244 \\
\hline & 5 & 0.9 & 0.01339 & 0.01525 & 0.01287 & 0.01302 & 0.01714 & 0.00242 & 0.00249 & 0.00240 & 0.00241 & 0.00240 & 0.00260 & 0.00273 & 0.00257 & 0.00258 & 0.00269 \\
\hline
\end{tabular}




\section{ESTIMATION FROM SAMPLES IN EXPONENTIAL \& GAMMA MODELS}

Table 10 (contd.). Exponential distribution empirical sample characteristic of reliability estimates in left censored samples

\begin{tabular}{|c|c|c|c|c|c|c|c|c|c|c|c|c|c|c|c|c|c|}
\hline \multirow[b]{2}{*}{$n$} & \multirow[b]{2}{*}{$r$} & \multirow[b]{2}{*}{$R(x)$} & \multicolumn{5}{|c|}{ BIAS } & \multicolumn{5}{|c|}{ VAR } & \multicolumn{5}{|c|}{ MSE } \\
\hline & & & MLE & MET. I & MET. II & MET. III & BLUE & MLE & MET. I & MET. II & MET. III & BLUE & MLE & MET. I & MET. II & MET. III & BLUE \\
\hline \multirow{6}{*}{8} & 1 & 0.9 & .01136 & 0.01137 & 0.01133 & 0.01133 & 0.01145 & 0.00170 & 0.00170 & 0.00170 & 0.00170 & 0.00176 & 0.00183 & 0.00183 & 0.00183 & 0.00183 & 0.00190 \\
\hline & 2 & 0.9 & 01422 & 0.01428 & 0.01414 & 0.01414 & 0.01219 & 0.00192 & 0.00192 & 0.00191 & 0.00191 & 0.00179 & 0.00212 & 0.00212 & 0.00211 & 0.00211 & 0.00194 \\
\hline & 3 & 0.9 & 0.01206 & 0.01224 & 0.01190 & 0.01191 & 0.01291 & 0.00168 & 0.00169 & 0.00168 & 0.00168 & 0.00174 & 0.00183 & 0.00184 & 0.00182 & 0.00182 & 0.00190 \\
\hline & 4 & 0.9 & 0.01117 & 0.01163 & 0.01092 & 0.01095 & 0.01395 & 0.00183 & 0.00184 & 0.00182 & 0.00182 & 0.00186 & 0.00195 & 0.00198 & 0.00194 & 0.00194 & 0.00205 \\
\hline & 5 & 0.9 & 0.01224 & 0.01346 & 0.01187 & 0.01192 & 0.01382 & 0.00182 & 0.00185 & 0.00181 & 0.00181 & 0.00191 & 0.00197 & 0.00203 & 0.00195 & 0.00195 & 0.00210 \\
\hline & 6 & 0.9 & 01170 & 0.01494 & 0.01121 & 0.01134 & 0.01492 & 0.00205 & 0.00216 & 0.00204 & 0.00204 & 0.00205 & 0.00219 & 0.00238 & 0.00216 & 0.00217 & 0.00228 \\
\hline \multirow{7}{*}{9} & 1 & & 01178 & 0.01179 & 0.01176 & 0.01176 & 0.01159 & 0.00144 & 0.00144 & 0.00144 & 0.00144 & 0.00160 & 0.00158 & 0.00158 & 0.00158 & 0.00158 & 0.00173 \\
\hline & 2 & 0.9 & 0.00997 & 0.01001 & 0.00991 & 0.00991 & 0.01143 & 0.00134 & 0.00134 & 0.00134 & 0.00134 & 0.00162 & 0.00144 & 0.00144 & 0.00144 & 0.00144 & 0.00175 \\
\hline & 3 & 0.9 & 0.01244 & 0.01255 & 0.01233 & 0.01233 & 0.01095 & 0.00157 & 0.00157 & 0.00156 & 0.00156 & 0.00155 & 0.00172 & 0.00173 & 0.00172 & 0.00172 & 0.00167 \\
\hline & 4 & 0.9 & 0.01109 & 0.01136 & 0.01091 & 0.01091 & 0.01175 & 0.00149 & 0.00149 & 0.00148 & 0.00148 & 0.00155 & 0.00161 & 0.00162 & 0.00160 & 0.00160 & 0.00169 \\
\hline & 5 & 0.9 & 0.01030 & 0.01090 & 0.01004 & 0.01006 & 0.01124 & 0.00151 & 0.00153 & 0.00151 & 0.00151 & 0.00141 & 0.00162 & 0.00165 & 0.00161 & 0.00161 & 0.00154 \\
\hline & 6 & 0.9 & 0.01098 & 0.01233 & 0.01061 & 0.01067 & 0.01273 & 0.00165 & 0.00169 & 0.00164 & 0.00165 & 0.00165 & 0.00178 & 0.00184 & 0.00176 & 0.00176 & 0.00181 \\
\hline & 7 & 0.9 & 0.01096 & 0.01436 & 0.01047 & 0.01063 & 0.01405 & 0.00172 & 0.00182 & 0.00171 & 0.00171 & 0.00194 & 0.00184 & 0.00203 & 0.00182 & 0.00183 & 0.00214 \\
\hline \multirow{8}{*}{10} & 1 & 0.9 & 0.01025 & 0.01026 & 0.01024 & 0.01024 & 0.00879 & 0.00128 & 0.00128 & 0.00128 & 0.00128 & 0.00121 & 0.00139 & 0.00139 & 0.00139 & 0.00139 & 0.00129 \\
\hline & 2 & 0.9 & 0.01030 & 0.01032 & 0.01026 & 0.01026 & 0.00821 & 0.00128 & 0.00128 & 0.00127 & 0.00127 & 0.00123 & 0.00138 & 0.00138 & 0.00138 & 0.00138 & 0.00130 \\
\hline & 3 & 0.9 & 0.00974 & 0.00981 & 0.00966 & 0.00966 & 0.00946 & 0.00132 & 0.00132 & 0.00132 & 0.00132 & 0.00128 & 0.00142 & 0.00142 & 0.00141 & 0.00141 & 0.00137 \\
\hline & 4 & 0.9 & 0.00902 & 0.00918 & 0.00888 & 0.00889 & 0.00971 & 0.00137 & 0.00137 & 0.00137 & 0.00137 & 0.00131 & 0.00145 & 0.00146 & 0.00144 & 0.00144 & 0.00141 \\
\hline & 5 & 0.9 & 0.01030 & 0.01064 & 0.01009 & 0.01011 & 0.00963 & 0.00136 & 0.00136 & 0.00135 & 0.00135 & 0.00126 & 0.00146 & 0.00148 & 0.00145 & 0.00145 & 0.00135 \\
\hline & 6 & 0.9 & 0.00979 & 0.01050 & 0.00952 & 0.00954 & 0.01009 & 0.00145 & 0.00147 & 0.00144 & 0.00145 & 0.00121 & 0.00155 & 0.00158 & 0.00154 & 0.00154 & 0.00131 \\
\hline & 7 & 0.9 & 0.01081 & 0.01226 & 0.01042 & 0.01048 & 0.01022 & 0.00148 & 0.00151 & 0.00147 & 0.00147 & 0.00142 & 0.00159 & 0.00166 & 0.00158 & 0.00158 & 0.00152 \\
\hline & 8 & 0.9 & 0.00903 & 0.01253 & 0.00856 & 0.00870 & 0.01249 & 0.00148 & 0.00157 & 0.00147 & 0.00148 & 0.00170 & 0.00157 & 0.00173 & 0.00155 & 0.00155 & 0.00186 \\
\hline
\end{tabular}

